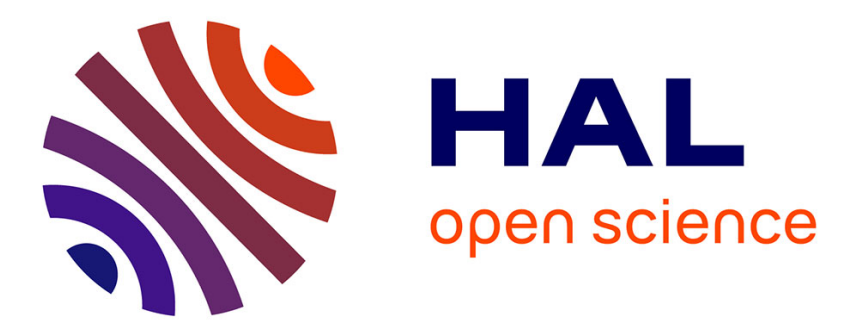

\title{
Performance of the Advanced GAmma Tracking Array at GANIL
}

\author{
J. Ljungvall, R.M. Pérez-Vidal, A. Lopez-Martens, C. Michelagnoli, E. \\ Clément, J. Dudouet, A. Gadea, H. Hess, A. Korichi, M. Labiche, et al.
}

\section{- To cite this version:}

J. Ljungvall, R.M. Pérez-Vidal, A. Lopez-Martens, C. Michelagnoli, E. Clément, et al.. Performance of the Advanced GAmma Tracking Array at GANIL. Nucl.Instrum.Meth.A, 2020, 955, pp.163297. 10.1016/j.nima.2019.163297 . hal-02440036

\section{HAL Id: hal-02440036 https://hal.science/hal-02440036}

Submitted on 13 Nov 2020

HAL is a multi-disciplinary open access archive for the deposit and dissemination of scientific research documents, whether they are published or not. The documents may come from teaching and research institutions in France or abroad, or from public or private research centers.
L'archive ouverte pluridisciplinaire HAL, est destinée au dépôt et à la diffusion de documents scientifiques de niveau recherche, publiés ou non, émanant des établissements d'enseignement et de recherche français ou étrangers, des laboratoires publics ou privés. 


\title{
Performance of The Advanced GAmma Tracking Array (AGATA) at GANIL
}

\author{
J. Ljungvall ${ }^{\mathrm{a}, *}$, R.M. Pérez-Vidal ${ }^{\mathrm{b}}$, A. Lopez-Martens ${ }^{\mathrm{a}}$, C. Michelagnoli ${ }^{\mathrm{c}, \mathrm{d}}$, E. Clément $^{\mathrm{c}}$, A. Gadea ${ }^{\mathrm{b}}$, A. Korichi $^{\mathrm{a}}$, M. Labiche $^{\mathrm{k}}$, N.
} Laloviće $^{\mathrm{e}}$, H. J. Li ${ }^{\mathrm{c}}$, F. Recchia ${ }^{\mathrm{h}}$, and the AGATA collaboration

\author{
${ }^{a}$ CSNSM, Université Paris-Sud, CNRS/IN2P3, Université Paris-Saclay, 91405 Orsay, France \\ ${ }^{b}$ Instituto de Física Corpuscular, CSIC - Universidad de Valencia, E-46980 Paterna, Valencia, Spain \\ ${ }^{c}$ GANIL, CEA/DRF-CNRS/IN2P3, BP 55027, 14076 Caen cedex 5, France \\ ${ }^{d}$ Institut Laue-Langevin, B.P. 156, F-38042 Grenoble cedex 9, France \\ ${ }^{e}$ Department of Physics, Lund University, SE-22100 Lund, Sweden

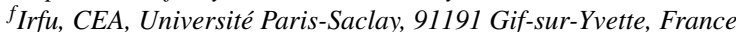 \\ ${ }^{g}$ IKP, University of Cologne, D-50937 Cologne, Germany \\ ${ }^{h}$ INFN Sezione di Padova and Dipartimento di Fisica e Astronomia dell'Universita', I-35131 Padova, Italy \\ ${ }^{i}$ Université de Strasbourg, CNRS, IPHC, UMR 7178, F-67000 Strasbourg, France \\ jInstitut de Physique Nucléaire de Lyon, Université de Lyon, Université Lyon 1, CNRS-IN2P3, F-69622 Villeurbanne, France \\ ${ }^{k}$ STFC Daresbury Laboratory, Daresbury, Warrington WA4 4AD, United Kingdom
}

\begin{abstract}
The performance of the Advanced GAmma Tracking Array (AGATA) at GANIL is discussed, on the basis of the analysis of source and in-beam data taken with up to 29 segmented crystals. Data processing is described in detail. The performance of individual detectors are shown. Efficiency of the individual detectors as well as efficiency after $\gamma$-ray tracking are discussed. Recent developments of $\gamma$-ray tracking are also presented. The experimentally achieved peak-to-total is compared with simulations showing the impact of back-scattered $\gamma$ rays on the peak-to-total in a $\gamma$-ray tracking array. An estimate of the achieved position resolution using the Doppler broadening of in-beam data is also given.

Angular correlations from source measurements are shown together with different methods to take into account the effects of $\gamma$-ray tracking on the normalization of the angular correlations.
\end{abstract}

Keywords: keywords

\section{Introduction}

In order to perform $\gamma$-ray spectroscopy nuclear structure studies in the conditions of extreme neutron/proton asymmetry and/or extreme angular momentum the so-called $\gamma$-ray tracking 5 arrays are considered as indispensable tools. Two international collaborations, AGATA (Advanced-GAmma-Tracking-Array) [1] in Europe and GRETA (Gamma-Ray Energy Tracking Array) in the US [2] are presently building such arrays. Position sensitive High-Purity Germanium (HPGe) detectors will cover 10 close to $4 \pi$ of solid angle and track the path of the $\gamma$ rays inside the detector medium giving maximum efficiency an excellent energy resolution. The technique of $\gamma$-ray tracking allows both the high efficiency needed for high-fold coincidences and the excellent position resolution needed for Doppler Correction at 15 in-flight fragmentation facilities such as the NUSTAR experiment at FAIR or FRIB.

Gamma-ray tracking starts from the digitally recorded waveforms, from the highly-segmented HPGe detectors, that are treated with Pulse Shape Analysis (PSA) techniques in order to extract the position of the interaction points of the $\gamma$ ray in the detector, presently with a position resolution of about $5 \mathrm{~mm}$ [3. 4]. The interaction points (hits) are grouped into events on

*Corresponding author: joa.ljungvall@ csnsm.in2p3.fr the basis of their timestamp, i.e. the absolute time of the $\gamma$-ray interaction. Different $\gamma$ rays in the same event and their ordered path are reconstructed from the hits via tracking algorithms. A higher efficiency with a high peak-to-total is expected as the solid angle taken by Anti-Compton shields is now occupied by HPGe crystals and the Compton event suppression is performed by the $\gamma$-ray tracking algorithm. The use of digital electronics allows a higher count-rate with maintained energy resolution, and rates up to $50 \mathrm{kHz}$ per crystals are routinely used during experiments. The continuous distribution of $\gamma$-ray interaction points that allows for the excellent Doppler correction seen in $\gamma$-ray tracking arrays also opens up and new degree of sensitivity in the determination of nuclear structure observables such as electromagnetic moments (e.g. lifetimes measurements based on Doppler shift and perturbed angular correlations). This paper is meant as both a snapshot in time of the capacities of AGATA and as a reference paper to be used when analysing data from AGATA experiments performed at GANIL.

The first experimental campaign with the demonstrator AGATA sub-array was at LNL [5] where it was coupled to the PRISMA spectrometer for the study of neutron-rich nuclei produced in fusion-fission and neutron-transfer reactions. This was followed by a campaign at GSI. Here a larger AGATA sub-array was coupled to the FRS separator [6] for the first campaign with radioactive ion beams. Performance of the AGATA sub-array 
at GSI has been extensively studied [7], with focus on the efficiency of the AGATA sub-array as a function of energy and data treatment. Other performance aspects such as the peak-to-total ${ }_{105}$ ratio was also investigated. The same set of data has been used to characterise the performance of AGATA using methods used with GRETINA [8] with a determined efficiency that deviates with $4.7 \sigma$.

${ }_{55}$ Since 2015, AGATA is installed [9] at GANIL, Caen, France. It is coupled to VAMOS (a variable mode high acceptance spectrometer) [10, 11]. Three measurement campaigns have been performed with focus mainly on neutron-rich nuclei populated using multi-nucleon transfer reactions or via fusion-fission or 60 induced fission. During 2018 a campaign where AGATA was coupled to the NEDA [12] neutron detector and DIAMANT [13, 14] charged particle detector was performed. A campaign of source measurements was performed during 2016 to, together with in-beam data, quantify the performance of AGATA

65 at the GANIL site as well. Basic performance data such as efficiencies are needed to analyze the data taken during the campaigns but a careful follow-up of the evaluation of the AGATA performance as the size of the array changes, detectors and electronics age and/or are changed is also of considerable interest.

70 It allows to assure that the performance is in accordance with what is expected. Furthermore, it helps understand where efforts to improve are important - this both at fundamental level, e.g. Pulse-shape analyses or $\gamma$-ray tracking, and on a more practical level learning how to best maintain the system at a high

75 level of performance. Extensive Monte Carlo simulations of AGATA are performed as well in order to predict the performance in different experimental configurations and with different number of AGATA crystals a thorough evaluation of the performance of such a detection system allows for the benchso marking of the Monte Carlo simulations, further helping the analysis of experimental data.

In this paper we will describe the performance of AGATA as of mid 2016, when it was equipped with 29 crystals. In section ${ }^{110}$ 2 and section 3 the experimental set up and data acquisition is

85 described. The performance of individual crystals is discussed in section 4 In section 5 the performance of AGATA as an array is discussed, using the Orsay Forward Tracking algorithm. Estimates of the position resolution achievable in a typical experiment are given in section 6 As the angular coverage of AGATA 90 increases the capabilities in terms of measuring angular correlations increase and this is discussed in section 7 Conclusions are given in section 8 .

\section{Experimental setup and data taking}

The present AGATA array consisted of 10 triple clusters 95 (Agata Triple Cluster, or ATC) [15] and one double cluster (Agata Double Cluster or ADC) arranged as schematically represented in figure 1 . Two of the detectors present in the frame ${ }_{120}$ where not connected to an electronics channel, giving a total of 30 active detectors. One detector showed varying performance,

100 related to used electronics, and is excluded form efficiency determinations. Measurements were performed both at what is referred to as "nominal position" i.e. the center of the AGATA $_{125}$ positioned at $23.5 \mathrm{~cm}$ from the target position, and at "compact position" with a distance of $13.47 \mathrm{~cm}$ between the closest part of the imaginary sphere that touches the front of the AGATA crystals and the target position. Different standard radioactive

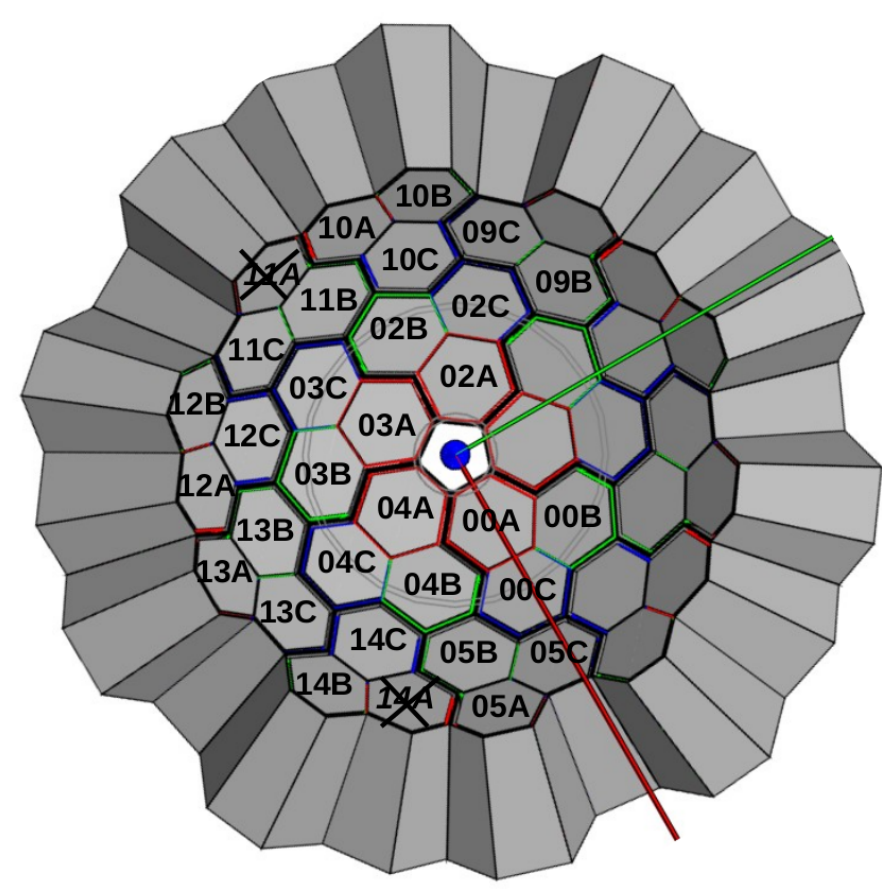

Figure 1: AGATA detectors seen from the reaction chamber point of view, labeled according to their position in the honeycomb. The two crossed over detectors are physically present but not connected to an electronic channel. Positions with no labeling are empty.

sources were placed at target position, see Tab. 1. Aluminum surrounding the target position, i.e. reaction chamber and target holder, were the same as in most of the experimental setups of the campaign. These aluminum structures are included in the Geant4 simulations [16, 17] presented in this work.

\begin{tabular}{|l|l|l|}
\hline Source & Activity [Bq] & $\mathrm{t}_{0}$ \\
\hline${ }^{152} \mathrm{Eu}$ & 22296 & $17 / 01 / 2013$ \\
\hline${ }^{60} \mathrm{Co}$ & 19632 & $21 / 08 / 2002$ \\
\hline
\end{tabular}

Table 1: Radioactive sources used for the measurement

For each detector, the data were collected from the 36 segments as well as for two different gains of the central contact (ranges of $\approx 8$ and $20 \mathrm{MeV}$ ). The segment signals are referred 15 to with a letter A-F and a number 1-6 where the letter gives the sector of the crystal and the number the slice, i.e. the segmentation orthogonal to the bore hole for the central contact. The AGATA raw data for each crystal in an event consist of, per each segment and for the central contact, the amplitude extracted from a trapezoidal filter with a, for the source data used in this paper, shaping time of $10 \mu \mathrm{s}$ as well as 100 samples (10 ns time between samples, $\approx 40$ pre-trigger and $\approx 60$ post-trigger) of the rise-time of the waveform and a time-stamp, used for the event building. The online and offline data processing is done using the same computer codes, and are described in detail in 
section 3

The preamplifier outputs were digitized and pre-processed by two different generation of electronics. The ATCA phase 1 electronics was developed at an early stage of the project for the AGATA Demonstrator, described in Ref. [1]. For the GANIL Phase a second generation of electronics was developed referred to as the GGP's [18]. The two generations of electronics use the same algorithms for determining the energy. However, for the determination of the time of a signal, the ATCA phase 1 electronics use a digital constant fraction whereas the GGPs use a low-level leading edge algorithm. These times are used for triggering purposes only, for both electronics digital ${ }_{190}$ CFDs are used for proper timing when analysing the data. A time signal is also extracted directly from the digitizer in order to provide a $\gamma$-ray trigger for the VME electronics of VAMOS.

\section{Data processing}

The raw data (event-by-event amplitude, timestamp and traces for segments and central contact) are treated with the chain of Narval actors as depicted in figure 2. Starting at the top we have data coming from the front-end electronics into20 the computer farm with the first Narval actor, the "Crystal Producer" that puts the data of the crystal into the Agata Data Flow. In the case of offline analysis the crystal data is read from disk. The next step, done in the "Preprocessing Filter" is to perform energy calibrations, time alignments, cross-talk205 corrections and the reconstruction of data in single crystal from an eventually missing segment (in case of several missing segments this is no longer possible). Following the preprocessing is the pulse-shape analysis where the $\gamma$-ray interaction positions are extracted using an adaptive grid search algorithm [19].210 Tests allowing to search for more than one interaction per segment of an AGATA crystal have been performed but the search is presently limited to one interaction per segment. From this point on the traces are removed from the data flow. In a typical experiment the result from the PSA is also written to disk $\mathrm{k}_{215}$ at this point as this allows redoing the subsequent steps in the analysis offline without the time consuming PSA. The final step where the data from each crystal is treated individually (Local Level Processing) is the "Post PSA", in which, apart from timestamp realignments, several energy correction procedure 220 described later are performed. After this, data from all AGATA crystals are merged in the "Event Builder" on the basis of a coincidence condition using the individual time stamps of each crystal. This is the start of what is referred to as the Global

170 Level Processing. Complementary detectors are added into the Agata Data Flow in the "Event Merger". This is done before 225 $\gamma$-ray tracking because complementary data from these detector, e.g. data form a beam tracking detector in case of a very large beam spot, can be needed for the tracking of the $\gamma$ rays.

175 Finally $\gamma$-ray tracking is performed. In this work the OFT $\gamma$ ray tracking algorithm has been used [20]. Finally the data is 230 written to disk by a "Consumer". This procedure is performed online for monitoring of the experiments (data processing) but also performed as a part of data analysis (data replay) starting online pulse-shape analysis. This possibility to also store the experimental traces to disk depends on the experimental conditions, and is in practice only possible if the number of validated events is lower than about $3 \mathrm{kHz}$ for 15 crystals and a bit less for 30 crystals. Automatic procedures have been developed, both for energy calibration purposes and for the preparation of the configuration files that the actors use allowing error free and fast analyses of experimental data.

\section{Crystal Performance}

In this section the performance in terms of energy and resolution for each crystal is discussed, named with their position in the AGATA frame at GANIL. The performance of the individual detectors were determined using measurements with ${ }^{60} \mathrm{Co}$ and ${ }^{152} \mathrm{Eu}$ sources, see table 1 . A set of standard procedures are performed to minimize the FWHM for each crystal. These procedures consist of cross-talk corrections and neutrondamage correction. The energies for events with more than one net-charge segments have to be corrected for cross talk, mainly between the central contact and the segments, as the energy calibration is performed mainly with event with segment multiplicity 1 . Correction coefficients are extracted from source data either by looking at the shift of the full-energy peak made by the summing of segments in fold two events or by looking at the base-line shift in fold one events. This procedure has been described in detail by Bruyneel at al. [21]. The correction for the effects of the neutron damage on the detection of the $\gamma$ rays of interest has been performed following the theoretical approach described by Bruyneel and coauthors [22]. Two calibration coefficients per detector channels, used to correct for the electron and hole trapping, are determined. This is done using a grid search based minimization of the FWHM and the left tail of the peaks in the spectra for each channel, i.e. 37 per detector. In figure 3 the effect of the correction is shown for one detector. This correction is more important for the segments as they are more sensitive to hole trapping, but it is also done for the central contact, and it is thus important also when the sum energy of hits inside a crystal for an event is forced to the value measured by the central contact. This correction is particularly important for measurements of lifetimes via line-shape analysis techniques, where the symmetry of the detector response function is extremely important to minimize systematical errors in the lifetime determination.

\subsection{Energy resolution}

The energy resolution has been determined for each segment and central contact for the crystals in the array at the moment of taking source data (2016). After the exposition to fast neutrons produced in deep inelastic collisions, fission and fusion evaporation reactions in the first campaign at GANIL in 2015, several AGATA crystals were damaged by the charge traps created by neutron radiation damage in the Ge crystal. These traps are lattice defects that lead to a reduction of the charge collection efficiency which appears as a low energy tailing on the energy line shape (red line in figure 3 . In position sensitive $\mathrm{Ge}$ detectors, like the AGATA ones, it is possible to apply an empirical 


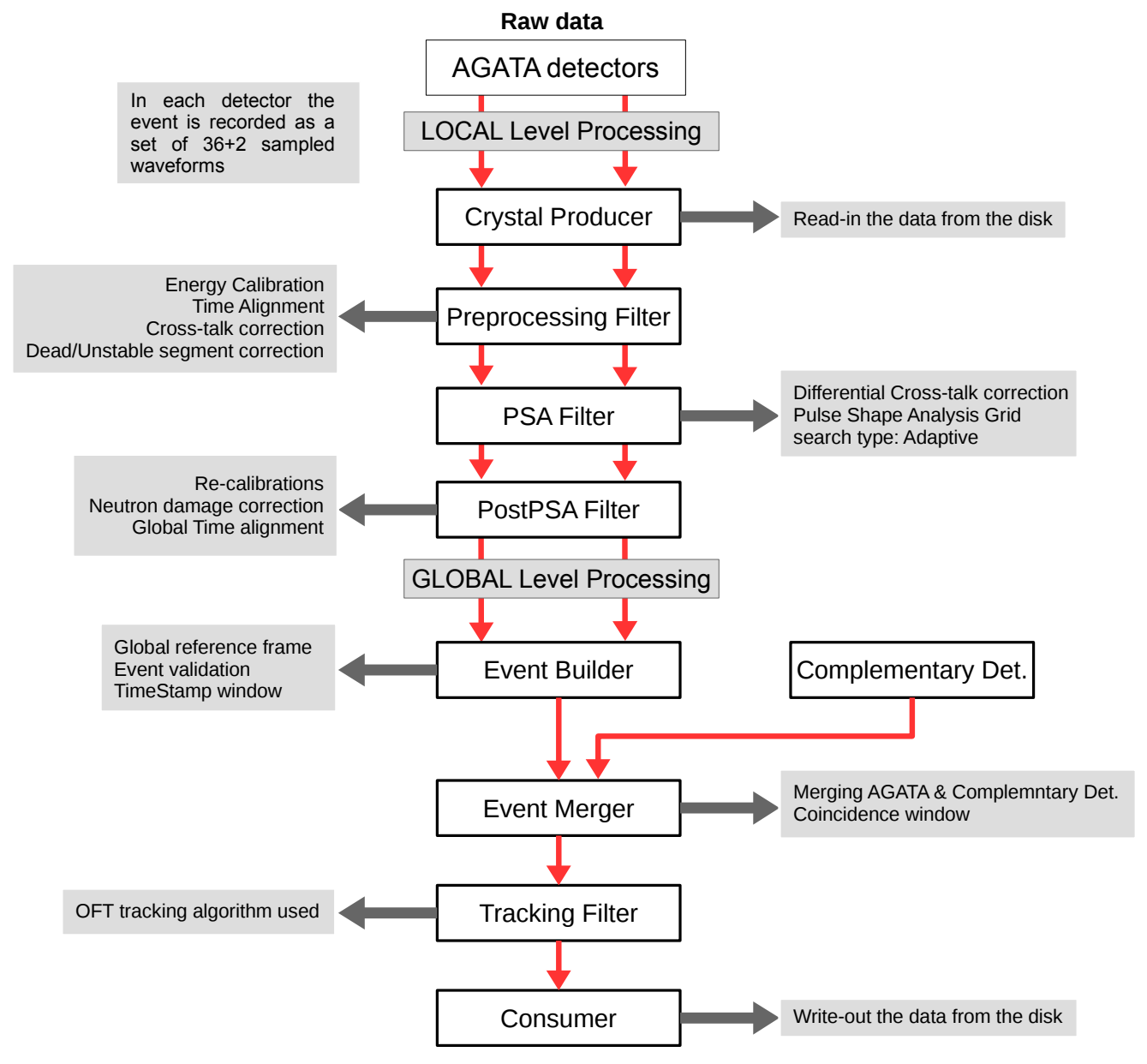

Figure 2: Chains of Narval actors used for data processing. For details see text.

correction to the neutron damage effects [22]. These correc-255 tions have been applied to the following 20 AGATA crystals in positions (with the corresponding crystal Id in parentheses): 00A(A001), 00B(B004), 00C(C010), 02C(C008), 03B(B002), 03C(C009), 09B(B011), 10A(A003), 10B(B003), 10C(C005), 11B(B013), 11C(C006), 12A(A002), 12B(B010), 12C(C001), 13A(A007), 13B(B007), 13C(C007), 14B(B001), 14C(C003). The original spectra and the ones after the corrections, for one 260 detector, are shown in figure 3 .

In figure 4 the resolutions for the central contacts and sum of segments for the used detectors are reported. The average FWHM resolution found for the central contacts before the neu-265 tron damage correction is $2.93 \mathrm{keV}$ and is improved to $2.57 \mathrm{keV}$ after neutron correction. In the case of the sum of segments the average FWHM is improved from $5.22 \mathrm{keV}$ to $3.08 \mathrm{keV}$, showing the difference in sensitivity to charge trapping. The comparison with the resolutions taken from detector data sheets 270 or factory measurements is reported in figure 5 In general all the measured FWHM resolutions for the crystals agree with the original ones, except for the detector 11C (B013) which apart from being neutron damaged had a resolution problem during the measurements, in both central contact and segments, due to problems with the electronics.

\subsection{Crystal efficiency}

The efficiency of each crystal has been determined first from the central contact signal. Although this is not the normal operation mode when performing $\gamma$-ray spectroscopy with AGATA, the crystal central contact efficiency allows easier diagnostic of the Data Acquisition Chain and easier comparison to Geant4 simulations. For these reasons, it is of great value. The early sub-arrays of AGATA, covering much less than $2 \pi$ of solid angle, can be used in nominal or in compact position, i.e. reducing the distance between the target or $\gamma$-ray source and the detectors by displacing the latter along the beam axis [9]. Two sets of data for efficiency measurement at the nominal and compact position of AGATA have been taken. All efficiency numbers quoted in this section are corrected for dead time of the data acquisition system. However, for the AGATA DAQ there is a general loss of about $10 \%$ of all events generated by the in- 


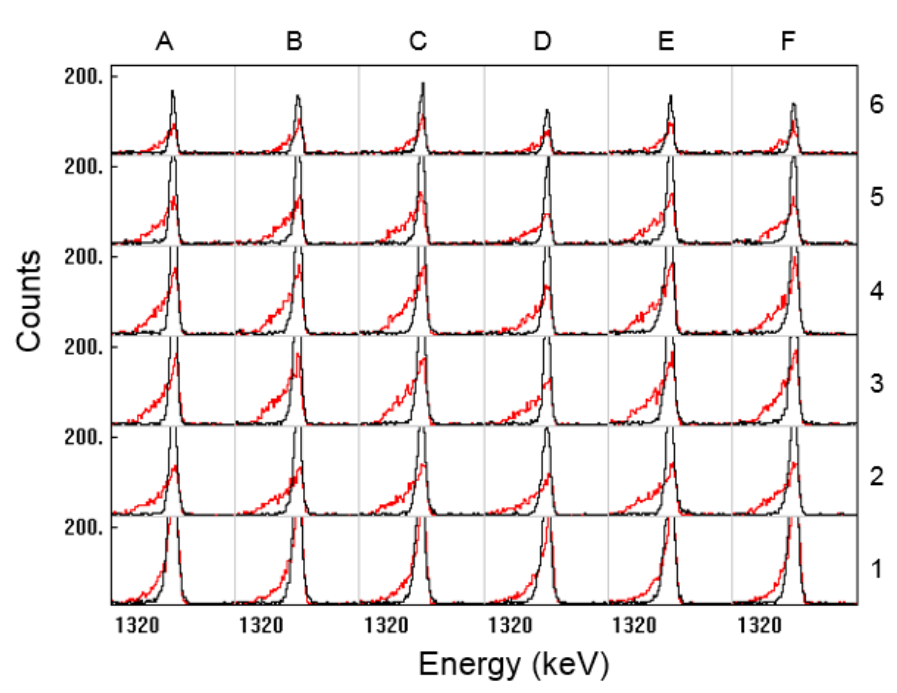

Figure 3: Example of the peak line shapes for the $1332 \mathrm{keV} 60 \mathrm{Co} \gamma$-ray before (red) and after (black) the neutron damage correction for the 36 segments of the crystal A002 position 12A ATC3.

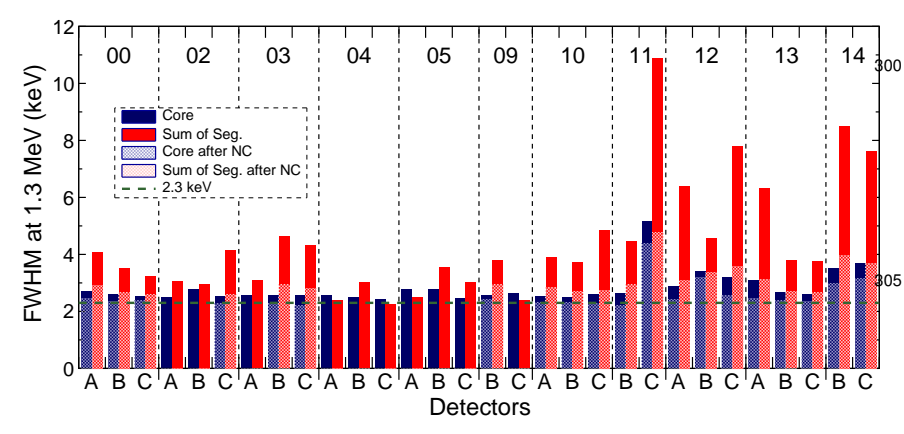

Figure 4: FWHM at $1.332 \mathrm{MeV}\left({ }^{60} \mathrm{Co}\right)$ of the central contact (blue) and the ${ }^{310}$ sum of segments (red) before (dark colours) and after (light colours) the neutron damage correction for the 30 capsules individually named with its position labels.

clusion the trigger processor that are used in experiments but ${ }_{315}$

corrected for in the numbers given below.

The efficiency has been determined both from $\gamma-\gamma$ coincidences, corrected for the angular correlation effects for the given geometry, and from singles central contact data taken with ${ }^{60} \mathrm{Co}$ and ${ }^{152} \mathrm{Eu}$ sources. The coincidence data are not af- 320 tral contact measurement is. To bypass this effect, the latter have been recorded in coincidence with the VME/VXI electronic of the GANIL acquisition system coupled to AGATA via the AGAVA board [1, 9]. The VME/VXI electronics dead time 325 dominates the dead time of the system and can easily be measured. The GANIL acquisition system is triggered by the OR of the AGATA digitizers CFDs, triggering the AGAVA board. The individual AGATA channels are then validated by the AGAVA request within a $300 \mathrm{~ns}$ time stamp coincidence window. At the 330 source rate, the VME/VXI GANIL electronic has a dead-time of $40 \mu s$ per read-out event, greater than the AGATA electronic system, and it can be precisely quantified and used for live time correction in the single central contact efficiency measurement.

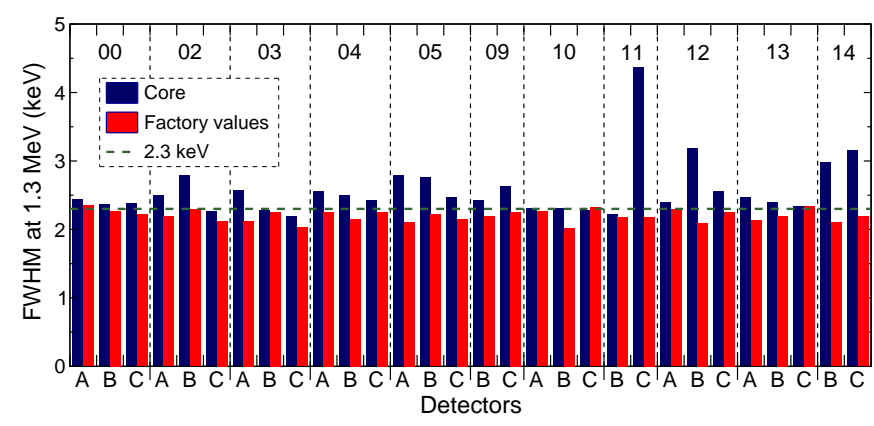

Figure 5: FWHM at $1.332 \mathrm{MeV}\left({ }^{60} \mathrm{Co}\right)$ of the central contact (red) after the neutron damage correction compared with the original FWHM measured by Canberra (blue) for the 30 capsules individually named with its position labels.

For the $\gamma-\gamma$ coincidence, the $1332 \mathrm{keV}-1173 \mathrm{keV}$ from the ${ }^{60} \mathrm{Co}$ source and $121.8 \mathrm{keV}-1408 \mathrm{keV}, 121.8 \mathrm{keV}-244.7 \mathrm{keV}$ and $344.3 \mathrm{keV}-778.9 \mathrm{keV}$ coincidence from the ${ }^{152} \mathrm{Eu}$ source were used. For fitting the $\gamma$-ray peak areas used to extract the efficiency values, the Radware software package was used [23]. A background subtraction was made by evaluating the correlated background on both sides of the gating energy for the $\gamma-\gamma$ coincidences analysis.

As a result of the measurements, the efficiency relative to a 3 in $\mathrm{X} 3$ in $\mathrm{NaI}$ detector (i.e., $1.210^{-3} \mathrm{cps} / \mathrm{Bq}$ at $25 \mathrm{~cm}$ ) for each detector at nominal position is reported in figure 6. In the same picture, the value at $1.3 \mathrm{MeV}$ as measured at the factory or during the customer acceptance tests is shown. The crystal in position $02 \mathrm{C}$ had electronic oscillation problems during the measurements and this is reflected in the lower efficiency as the detector was not functional for periods during the measurement. The average measured value is $79 \%$, close to the factory values average of $81 \%$.

The absolute central contact efficiency for the whole array, composed by 29 operational crystals, is reported in figure 7 for the nominal position of AGATA and figure 8 for the compact position of AGATA. Here each crystal is treated as a single detector like in a standard $\gamma$-ray detector array. The values obtained by the singles are compared with the $\gamma-\gamma$ results and simulations and overlap well. For the nominal geometry, the efficiency measured using singles is $2.95(6) \%$ at 1332 $\mathrm{keV}$ whereas for the compact geometry it is $5.5(1) \%$ at 1332 $\mathrm{keV}$. Geant4 simulations using the AGATA simulation package [17] have been performed with a realistic implementation of the reaction chamber used during the experimental campaign at GANIL [9]. There is $12 \%$ discrepancy between the simulation and the experimental results. This difference is larger than the $2.5 \%$ average discrepancy for the individual crystals, as shown in figure 6, between factory measurements and measurements made within the AGATA collaboration. However, Geant 4 simulations of the three differently shapes crystals used by AGATA give a relative efficiency of $86 \%, 86 \%$, and $87 \%$, for type $\mathrm{A}, \mathrm{B}$, and $\mathrm{C}$, respectively. The average measured value is $79 \%$, or $8 \%$ lower. This is in reasonable agreement with the $12 \%$ of efficiency missing when compared with simulation, as is illustrated in figures 7 and 8 (cross symbols) where the ef- 
of each crystal has been scaled to its measured value. Here the question of how the $12 \%$ of effective germanium is lost has to be raised. The presence of a dead layer or missing germanium will have an impact on the PSA as it is done under assumptions on the active volume and shape of the germanium dimulations assuming a thicker dear layers improve the correspondence with experimental data but it is difficult to pin down the contribution from different surfaces of the detectors, i.e., one can reproduce experimental data with different combination of dead layers around the central contact and at the back of the detector. Moreover, the mismatch of the efficiencies at low energies can not be corrected reducing the active volume around the central contact or at the back of the detector. In figures 7 and 8 simulations with dead layer of $2.5 \mathrm{~mm}$ around the central contact and $3 \mathrm{~mm}$ at the back are also shown.

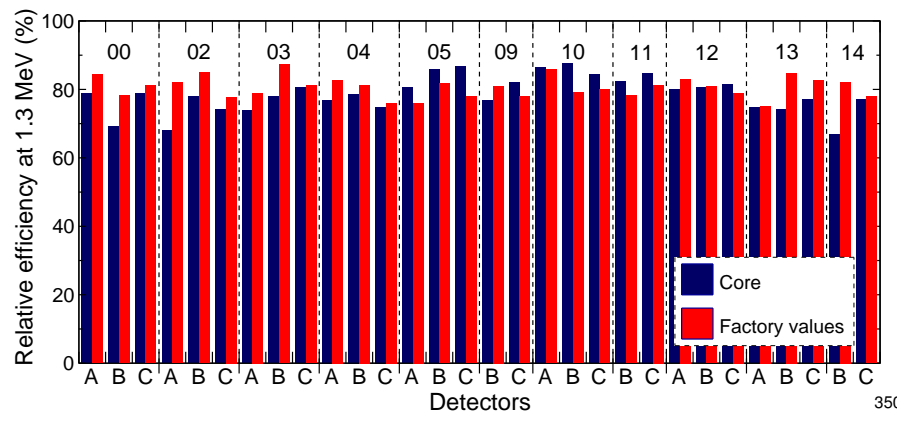

Figure 6: Relative central contact efficiency at $1.3 \mathrm{MeV}\left({ }^{60} \mathrm{Co}\right)$ in comparison with the initial relative efficiency as provided by manufacturer for the 29 capsules individually named with its position label.

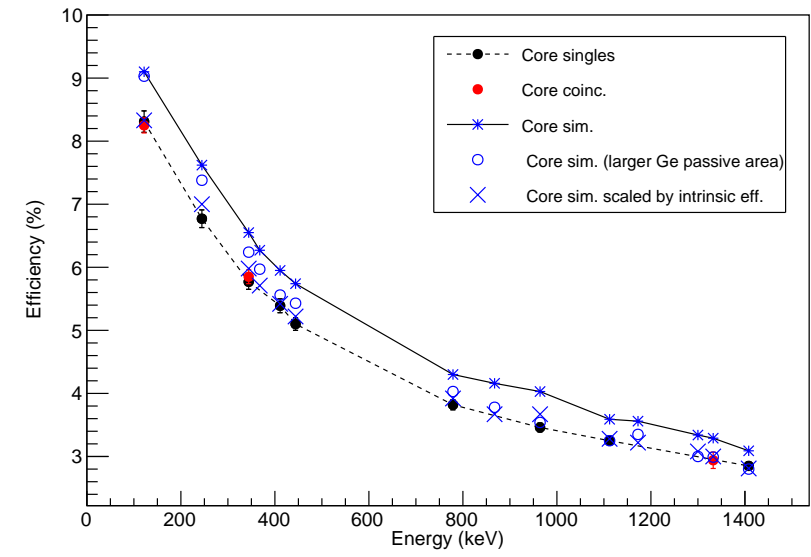

Figure 7: Absolute central contact efficiency for the 29 capsules AGATA subarray in nominal position $(23.5 \mathrm{~cm}$ distance source to detectors) obtained with the data from ${ }^{152} \mathrm{Eu}$ and ${ }^{60} \mathrm{Co}$ in singles (black circles) and in coincidences (red circles) in comparison with the simulations (black stars). The blue crosses corresponds to simulations where the efficiency has been scaled according to the difference between the simulated absolute efficiency and the measured absolute efficiency. Simulations performed with increased dead layers are also shown (Empty blue circles). See text for details. The rate per crystal at this position was around $200 \mathrm{~Hz}$. Error bars are smaller than used symbols.

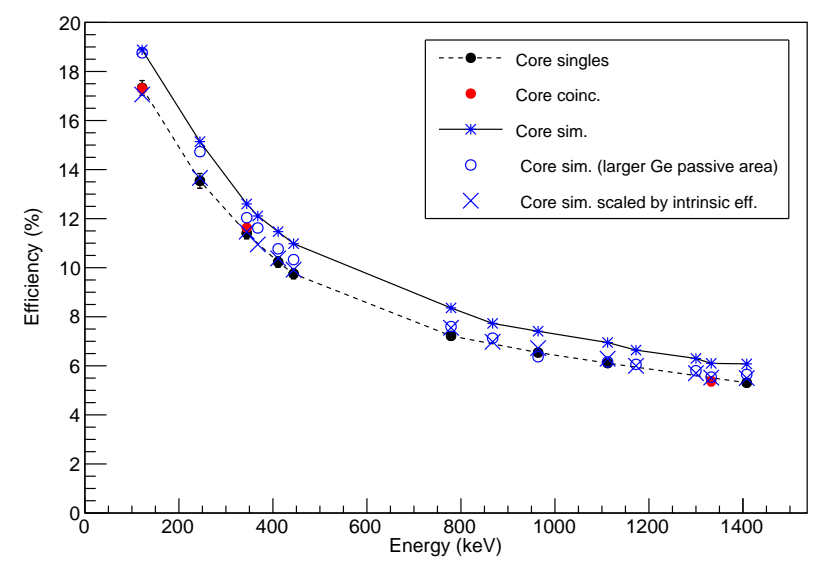

Figure 8: Absolute central contact efficiency for the 29 capsules AGATA subarray in compact position (13.47 cm distance source to detectors measured on the beam axis) obtained with the data from ${ }^{152} \mathrm{Eu}$ and ${ }^{60} \mathrm{Co}$ in singles (black circles) and in coincidences (red circles) in comparison with the simulations (black stars). The blue crosses corresponds to simulations where the efficiency has been scaled according to the difference between the simulated absolute efficiency and the measured absolute efficiency. Simulations performed with increased dead layers are also shown (Empty blue circles). See text for details. The rate per crystal at this position was between 300 and $500 \mathrm{~Hz}$. Error bars are smaller than used symbols.

\section{Performance of the AGATA array with the Orsay For- ward Tracking}

Description of OFT. The Orsay Forward Tracking (OFT) algorithm [20] was developed with simulated data sets produced with the Geant4 AGATA code [17]. The output of the simuditions, such as energy resolution and threshold and position resolution allowing the optimization of the algorithm using realistic input. As all forward-tracking algorithms the OFT starts with clustering interaction points. These clusters are evaluated using a $\chi^{2}$-like test where the angle between interaction points as given by the interaction positions given by the PSA are compared with the angles as given by the Compton scattering formula and energies in each interaction point. The best permutation for each cluster is calculated and the clusters are sorted in order of best figure of merit. Clusters that pass a threshold called $P_{\text {track }}$ are accepted as good $\gamma$ rays. The most influential paramter in this is $\sigma_{\theta}$ corresponding to the error in angle coming from the error in interaction positions from the PSA. Using simulations this parameter was optimized to $\sigma_{\theta}=0.24 \mathrm{~cm}$ corresponding to the assumed position resolution in the simulations of $5 \mathrm{~mm}$ at $100 \mathrm{keV}$ interaction point energy. Interaction points that are further away than $4 \mathrm{~cm}$ from the closest other interaction point are treated as a photo-electric absorption event. Here the probality for a $\gamma$ ray to have penetrated this deep and been aboserbed via the photo electric effect is evaluated and compared to the $P_{\text {sing }}$ parameter. The single-interaction-point evaluation is an important part of the tracking algorithm since the efficiency loss when it is not included is very large for low-energy events, and non negligible at higher energies: $\sim 20 \%$ of $1.4 \mathrm{MeV}$ total-absorption events in each individual detector are single in- 
teraction points. This last fact is due to the way Pulse Shape Analysis (PSA) identifies interaction points in the AGATA de-425 tectors. Indeed, up to now, the Grid Search algorithm [19] used online only looks for 1 interaction point per segment. This is at the fits of the segment traces allow for more than one hit per segment. For a detailed explatation on the OFT algorithms see ${ }_{430}$ Lopez-Martens et al. [20].

OFT parameters. The definition and typical ranges of the main parameters of OFT are summarised in table 2 .

Table 2: Table summarising the meaning and standard ranges of the main ad- ${ }_{435}$ justable parameters of OFT.

\begin{tabular}{|c|c|c|}
\hline parameter & definition & typical value \\
\hline$\sigma_{\theta}$ & $\begin{array}{c}\text { average interaction-point } \\
\text { position resolution }(\mathrm{cm})\end{array}$ & $0.3-3$ \\
\hline$P_{\text {sing }}$ & $\begin{array}{c}\text { minimum probability to accept } \\
\text { single-interaction-point clusters }\end{array}$ & $0.02-0.15^{440}$ \\
\hline$P_{\text {track }}$ & $\begin{array}{c}\text { minimum figure of merit to accept } \\
\text { mutliple-interaction-point clusters }\end{array}$ & $0.02-0.05$ \\
\hline
\end{tabular}

Tuning the parameters can affect the spectral quality and shape. As an example, a high value of $\sigma_{\theta}$ corresponds to nearly fully relaxing the comparison between scattered energies obtained from interaction positions and scattered energies obtained from energy differences. Basically, using a very large $\sigma_{\theta}$ reduces the cluster evaluation stage to finding the most likely sequence of interaction points in a cluster on the basis of ranges and interaction probabilities only. Increasing $\sigma_{\theta}$ increases the high-energy efficiency. However, it also decreases

400 the low-energy efficiency in the case of medium to high photonmultiplicity events since single-interaction points are being accepted as members of multi-interaction point clusters and are therefore lost as potential $\gamma$ rays absorbed in a single interaction. There is an optimal value of $\sigma_{\theta}$, which maximises the gain in efficiency at medium and high energy while minimizing the loss of efficiency at low energy. By analysing source and in-beam data obtained at Legnaro, GSI and GANIL, the optimal value of $\sigma_{\theta}$ is found to be around $\sim 0.6$ and $0.8 \mathrm{~cm}$. This corresponds to an average experimental position resolution a ${ }_{410}$ factor of 2 to 3 worse than anticipated. This is consistent with measurements of the position resolution of an interaction point as a function of the deposited energy [4] as well as with the observed clusterisation of interaction points in specific areas of the detector segments.

415 Another example is given by the energy range of the singleinteraction spectrum, which grows when the threshold for validation of the corresponding clusters is lowered. For $P_{\text {sing }}=0.15,450$ the spectrum extends to $\sim 600 \mathrm{keV}$, while for $P_{\text {sing }}=0.02$, it goes beyond $2 \mathrm{MeV}$. Extending the spectrum increases the overall efficiency at high-energy. The price to pay, however is a large background: for $P_{\text {sing }}=0.02$, the single-interaction points are responsible for nearly two thirds of the background present in the ${ }_{455}$ spectrum of tracked photon energies. Recent developments in the OFT code has improved on this points and uses an empirically deduced energy-"distance in germanium" relationship instead of the single parameter $P_{\text {sing }}$, allowing an improved peakto-total [25].

The optimal value of $P_{\text {track }}$ is found to be around 0.05. Some very slight adjustments can be made as a function of $\sigma_{\theta}$ but the general trend is that a smaller value leads to more background and a larger value reduces the peak intensities.

\subsection{Tracking Efficiency measurements}

The standard set of OFT parameters $\left(\sigma_{\theta}=0.8, P_{\text {track }}=0.05\right.$ and $P_{\text {sing }}=0.05$ ) were used to extract the tracking efficiency of AGATA at GANIL in a configuration with 29 capsules. The efficiencies to track the photons emitted by a ${ }^{152} \mathrm{Eu}$ source were obtained by comparing the detected peak areas to the expected intensities given the source activity, the measurement time interval and the electronics dead time. Since there are several 2-photon cascades in the radioactive decay of ${ }^{152} \mathrm{Eu}$, the efficiencies at certain photon energies can also be measured by comparing the detected peak area of a transition when a coincidence with the transition of interest is required or not. The advantage of this second method is that no knowledge of the source activity or dead time of the system is required. The efficiencies obtained are shown in figure 9

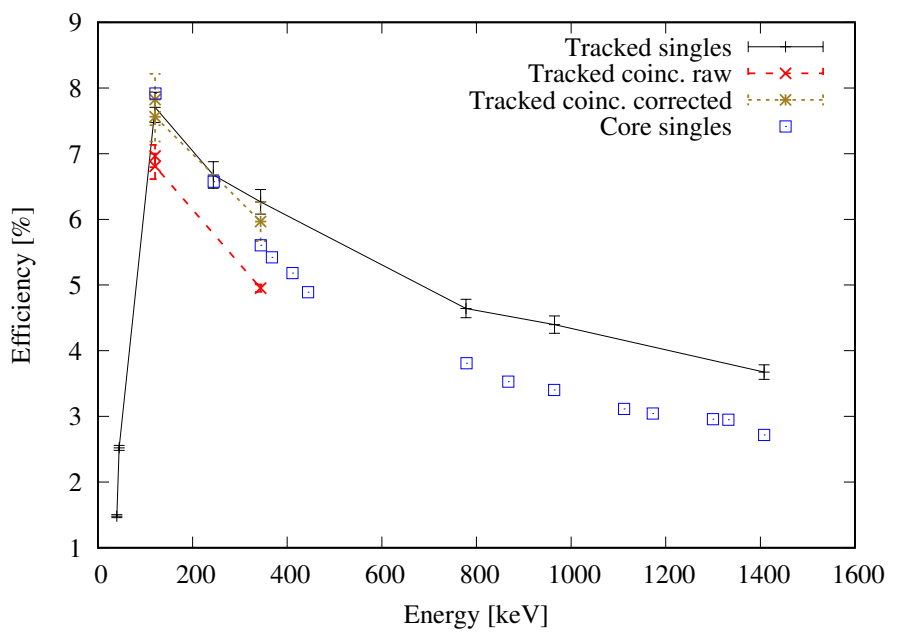

Figure 9: Tracking efficiency of 29 AGATA detectors as a function of photon energy obtained with the standard OFT parameter set and using either the total singles tracked spectrum or the (121 keV-244 keV), (121 keV - $1408 \mathrm{keV})$ and (344 keV - $778 \mathrm{keV}) \gamma-\gamma$ coincidences. The efficiency for 29 cores scaled from figure 7 is also shown. See text for details.

The efficiency to track a $1.4 \mathrm{MeV}$ photon with 29 capsules is found to be $3.67(1) \%$. This corresponds to an add back factor with respect to the efficiency of the 29 detectors taken individually of $1.285(4)$.

In figure 9, the raw coincidence efficiencies at 121 and 344 $\mathrm{keV}$ lie below the singles tracking efficiency curve. This is because the tracking efficiency varies with the angle between the emitted photons; most notably it vanishes for small angles due to the deficiencies of the AGATA PSA algorithm and/or due to the fact that the tracking algorithm cannot disentangle the 
points belonging to the 2 coincident photons arriving to close. This is clearly seen in the plot of the $\gamma-\gamma$ angular correlations 485 for the 121.8-244.7 and 344.3-778.9 coincidences in ${ }^{152} \mathrm{Sm}$ and

${ }^{152} \mathrm{Gd}$ shown in figure 10 By correcting the coincidence efficiencies by the missing fraction of the experimental angular correlations compared to the theoretical curve, the correct tracking efficiency values are recovered.
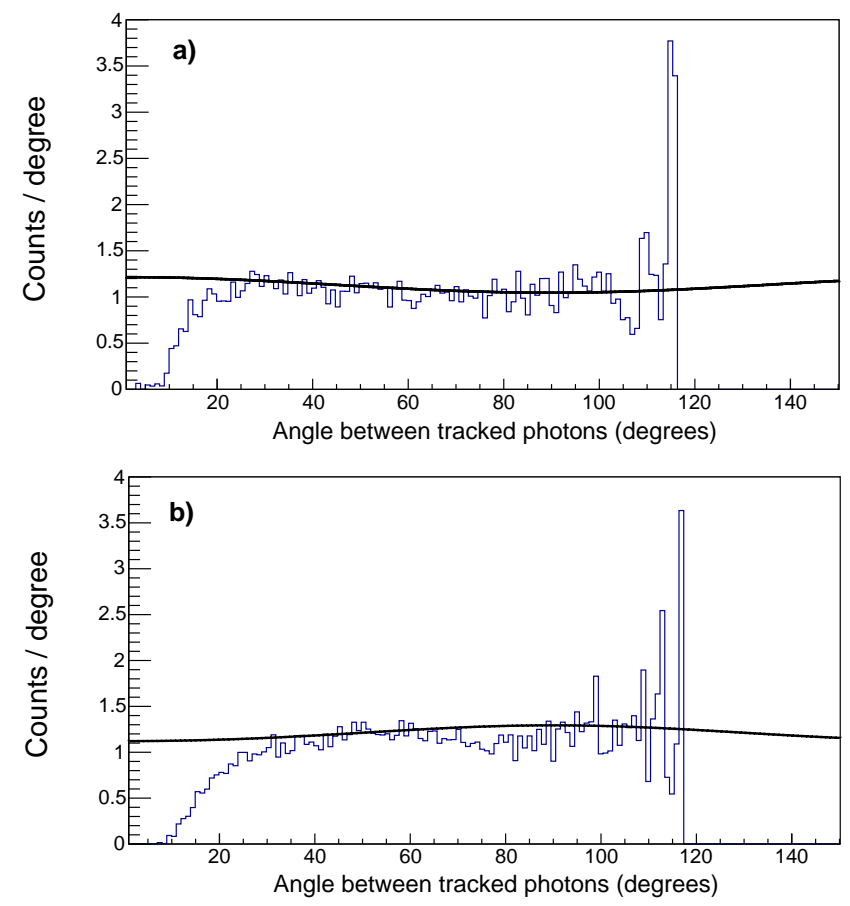

Figure 10: a) $\gamma-\gamma$ angular correlations obtained for the $121.8 \mathrm{keV}-244.7 \mathrm{keV}$ cascade in ${ }^{152} \mathrm{Sm}$ using the OFT parameter $\sigma_{\theta}=0.8$. b) same as a) for the 344.3 $\mathrm{keV}-778.9 \mathrm{keV}$ cascade in ${ }^{152} \mathrm{Gd}$ and in the case of $\sigma_{\theta}=2.0$. The solid lines represent the best adjustment of the theoretical curves to the data.

Using a larger value of $\sigma_{\theta}$ leads to a slightly lower tracking efficiency below $200 \mathrm{keV}$ but yields $13 \%$ more efficiency at $1.4 \mathrm{MeV}$, making the add back factor increase to $\sim 1.4$. It also changes the raw coincidence efficiencies for some coincidence couples. In the case of the $344.3 \mathrm{keV}-778.9 \mathrm{keV}$ cascade of figure 10, in particular, correlations are not only absent at small angles, but also at larger angles, when OFT most probably misinterprets all or a subset of the interaction points of the event as points belonging to a back-scatter sequence.

\subsection{Tracking Peak-to-Compton ratio}

An important performance parameter for a $\gamma$-ray spectrometer is the peak to total quantifying the fraction of events found in the full energy peak as compared to the total number of detected $\gamma$ rays. Data was taken with a ${ }^{60} \mathrm{Co}$ source with an activity of $8.7 \mathrm{kBq}$. Gamma-ray tracking was then performed offline for 29 of the 30 AGATA detectors using the 30th as an external trigger. In the 30th detector a central contact energy of $1332.5 \pm 5$ $\mathrm{keV}$ was demanded. In this manner a $\gamma$-ray multiplicity of one can be guaranteed for the remaining 29 detectors. In figure $11_{95}$ the $\gamma$-ray spectrum is shown, together with spectra made with two different treatments of single interaction validation. The peak-to-total using the empirically fitted maximum distance in germanium for single interactions is $36.4(4) \%$. It is well known that the peak-to-total in a $\gamma$-ray tracking array is dominated by single point interaction accepted as events with a direct absorption of the total $\gamma$-ray energy via the photoelectric effect. Excluding such events the peak-to-total is increased to $52.4(6) \%$, with a reduction in efficiency for the full energy peak of $17 \%$. The variation of peak-to-total and efficiency at $1173 \mathrm{keV}$ as a function of the $P_{\text {track }}$ is shown in figure 12 , for both the cases when single interactions are included or excluded.

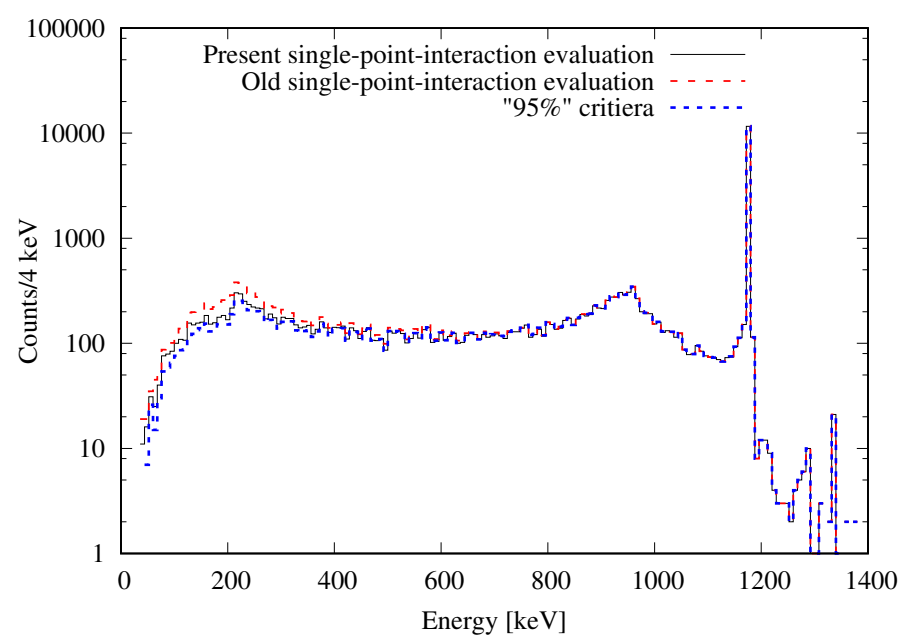

Figure 11: Gamma-ray tracked spectra for 29 AGATA detectors for ${ }^{60} \mathrm{Co}$ data using a 30th AGATA detector as a external trigger by demanding the full absorption of the $1332.5 \mathrm{keV}$ gamma in it. The solid line (black) is using the latest single-point interaction validation procedure, the dashed line (red) is using the old single-point interaction validation procedure, and finally the dotted line (blue) using the $95 \%$ absorption limit.

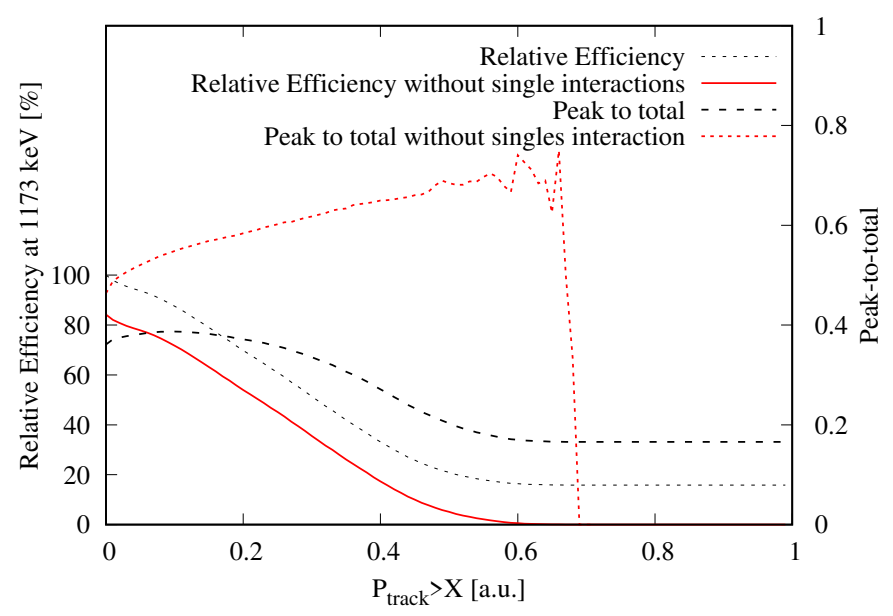

Figure 12: Efficiency relative to $P_{\text {track }}=0$ at $1173 \mathrm{keV}$ and peak to total as a function of $P_{\text {track }}$ used by the OFT algorithm to accept or not a $\gamma$-ray track. This for when including or excluding single point interactions.

Monte Carlo simulations using the AGATA simulations packages were made in order to compare simulated $\gamma$-ray track- 
ing performance with experimental data. In the simulations a ${ }^{60} \mathrm{Co}$ was simulated with a source strength of $5 \mathrm{kBq}$. An absolute time was used in the simulation allowing effects such as pile-up and random coincidences to be simulated. Gamma-ray interactions in the same segment were packed at their energyweighted average positions. These were then written into the same data format as used to store experimental post-PSA data. This allowed the use of identical $\gamma$-ray tracking and post treatment codes for the experimental and simulated data, i.e. the simulated data was treated exactly as explained for the experimental data above. Four different simulations were performed. The first one including only the HPGe crystals and the aluminum end-caps. The second simulation included a large piece of steel positioned to mimic the large quadrupole magnet of the VAMOS. The third simulation included both the large piece of steel mimicking VAMOS, concrete walls and the target chamber. A fourth simulation was also performed adding to the third simulations thicker dead layers to the HPGe crystals. The added dead layers were $3 \mathrm{~mm}$ at the coaxial end of the detector and $2.5 \mathrm{~mm}$ around the central contact. The peak-to-total for the different simulations, when gating on the $1332.5 \mathrm{keV}$ transition to look at the $1173 \mathrm{keV}$ transition were $49 \%, 48 \%, 43 \%$, and $41 \%$, respectively. This is to be compared to the experimental value of $36 \%$. In figure 13 the Compton scattering part of the $1332.5 \mathrm{keV}$ gated ${ }^{60} \mathrm{Co}$ spectra are shown for experimental and simulated data. In the experimental spectrum a pronounced back-scattering peak can be seen just above $200 \mathrm{keV} .555$ The simulation labeled 1, only including AGATA itself, does not show such a back-scattering peak. And consequently the peak-to-total is much better than for the experimental data. For simulation 2, where the VAMOS quadrupole has been included in a very schematic way a clear back-scattering peak emerges. ${ }^{560}$ However, at both lower and higher energies as compared to the back-scattering peak the experimental data contains more counts. In simulation 3 , where the concrete walls are included together with the scattering chamber a shape of the spectrum very close to the experimental one is produced. This suggests565 that a significant fraction of the spectrum is not due to Compton scattering inside the HPGe crystals of AGATA but from the scattering on the structures around AGATA into AGATA. Including thicker dead layers in the HPGe crystals in the simulation, as done for the fourth simulation, increases slightly570 the amount of background between the full-energy peak and the Compton but does not change the shape of the spectrum in a significant way. However, the peak-to-total is decreased by about 5\%. These "back scattered" $\gamma$ rays are very difficult to properly discriminate against as they from the point of view of 575 $\gamma$-ray tracking are perfectly good single interaction point events

545 in the front of the crystals.

\subsection{In-beam efficiency of AGATA coupled to VAMOS}

It is of considerable interest to measure the in-beam effi-580 ciency of AGATA as well, as the efficiency is also a function of count rate in the individual detectors due to pile-up (rejected and non rejected) and rate limitations in the electronics. The lack of detail in this section is voluntary as the actual in-beam efficiency is very experiment dependent and hence not of great ${ }_{585}$

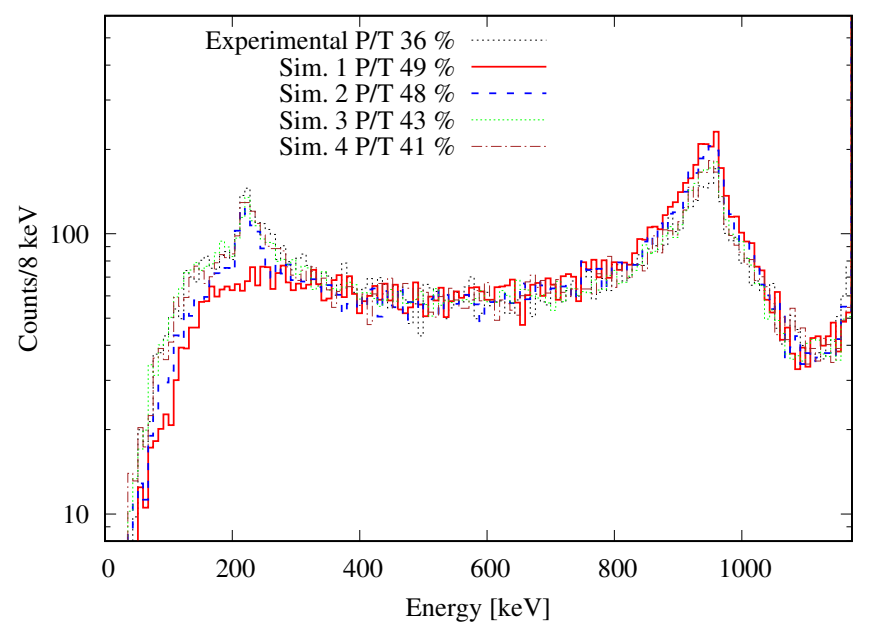

Figure 13: Comparisons between experimental spectrum (in black) and spectra from four different simulations. The spectra are normalized to the same number of counts in the region $0-1165 \mathrm{keV}$. Simulation 1 includes only AGATA, simulation 2 also includes a schematic implementation of the VAMOS dipole magnet, simulation 3 further adds concrete walls around the experimental setup. Finally, simulation 4 has thicker dead layers added to the HPGe crystals. For further details on the simulations, see the text.

general interest. The aim of the section is to give a useful rule of thumb to allow consistency checks when analysing data. The in-beam efficiency for events with a higher $\gamma$-ray fold than one also depends on the angular distribution of and correlation of the $\gamma$-ray transitions used to measure it. This both via pure geometrical effects and via the lowered $\gamma$-ray tracking efficiency for $\gamma$ rays with a preference for being emitted in parallel. The in-beam efficiency has been estimated for AGATA coupled to VAMOS for an experiment where a ${ }^{92}$ Mo beam impinged on a ${ }^{92}$ Mo target, and the beam-like reaction products were unambiguously identified in VAMOS, also providing the velocity vector for Doppler correction. During this experiment 23 AGATA crystals were operational in the array, each counting at around $45 \mathrm{kHz}$ with a shaping time of $2.5 \mu \mathrm{s}$. As the target and the beam both were ${ }^{92} \mathrm{Mo}$, de-excitation of target like and beam like particles could be studied. The beam-like and target-like nuclei travel with a relative angle of about $90^{\circ}$, allowing an estimate of the effect of the angular distribution on the measured efficiency.

The coincidence method was used to determine the efficiency at $1510 \mathrm{keV}$, i.e., the number of detected $2_{1}^{+} \rightarrow 0_{1}^{+} \gamma$ rays per detected $\gamma$ ray from the $4_{1}^{+} \rightarrow 2_{1}^{+}$was determined. Peak intensities were extracted from singles spectra and from $\gamma \gamma$ coincidence matrices. The singles spectrum and the projected gate in the $\gamma \gamma$ coincidence matrix are shown in figure 14. The efficiency at $1510 \mathrm{keV}$ extracted using this method is after $\gamma$-ray tracking $1.5(1) \%$, to be compared with the expected efficiency of about $2.5 \%$ for 23 AGATA crystals at an energy of $1.5 \mathrm{MeV}$. This loss of efficiency, some $40 \%$ lower, has several origins. At count rates of about $45 \mathrm{kHz}$ and a shaping time of $2.5 \mu \mathrm{s}$ there is a loss in the order of $20 \%$ points from pile up in the AGATA detectors [26]. There is also the loss in tracking efficiency for higher fold events. This can be estimated using the 

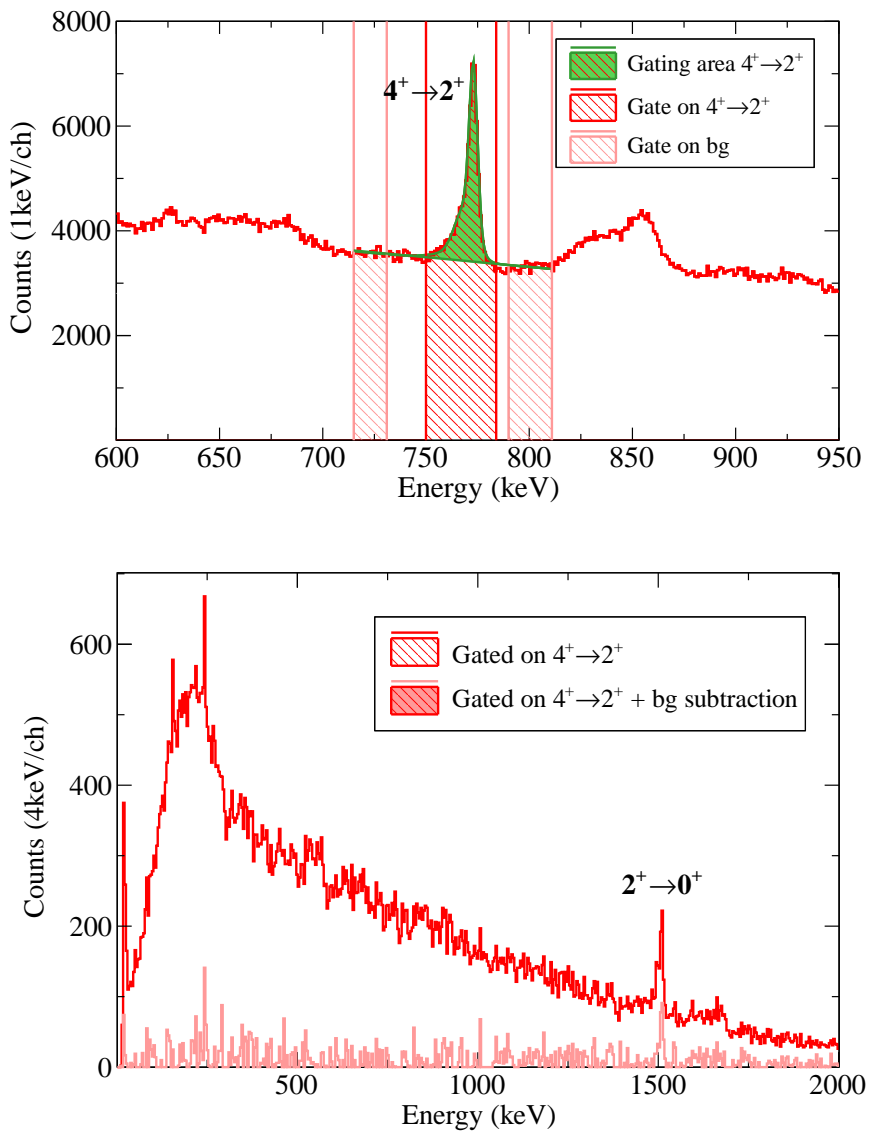

Figure 14: Top panel, singles $\gamma$-ray spectrum showing the gate set on the 773 $\mathrm{keV} 4_{1}^{+} \rightarrow 2_{1}^{+}$transition in ${ }^{92} \mathrm{Mo}$. Bottom panel, $\gamma$-ray spectra showing the $1510 \mathrm{keV} 2_{1}^{+} \rightarrow 0_{1}^{+}$transition used to estimate the in beam efficiency. smallest used cluster angle in the OFT of $8^{\circ}$ (as can be seen in figure 10 the efficiency to track two $\gamma$ rays inside this cone is close to zero) which corresponds to approximately $6 \%$ of the solid angle of 23 AGATA detectors. These contributions add up to about $25 \%$ points of losses that are rate dependant, via the pile up, and related to the detector physics (i.e. the rise time of the HPGe crystals and average cluster size for typical $\gamma$ rays) and therefore always will be present. There is an open question from where the remaining about $15 \%$ points of efficiency loss is coming. Measurements suggests that maybe $5 \%$ points could come from overload beyond specification of the trigger distribution system related to the high total rate (more than $1 \mathrm{MHz}$ ).

\section{Position resolution of the PSA}

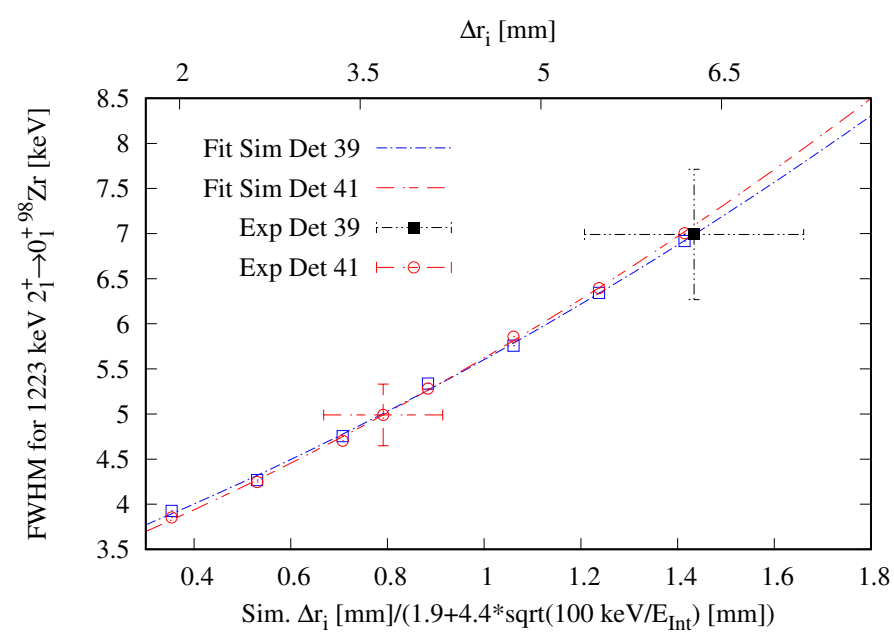

Figure 15: Estimated position resolution for two detectors in the AGATA array. The lines are fits to the FWHM of simulated data sets for the two different detectors where the assumed position resolution has been varied. Large symbols show the measured FWHM for each detector (y-axis) and corresponding deduced position resolution (x-axis). For details on simulation and experiment, see text. Note that the lower $\mathrm{x}$-axis is a scaling factor of the position resolution given in Söderström et. al. [4] for the FWHM in one dimension. The upper $\mathrm{X}$-axis shows the average resolution for the interaction point used for Doppler Correction.

The VAMOS allows for a very precise determination of the recoil vector of the identified ion. The direction can in this context be considered as exact whereas the velocity has an error in the order of a few per mill. Given that the recoil velocity has a very small error the position resolution can be estimated by the Doppler Broadening of the $\gamma$-ray peaks via the Doppler Shift given by

$$
E_{\gamma}=E_{\gamma 0} \frac{\sqrt{\left(1-\beta^{2}\right)}}{(1-\beta \cos \theta)}
$$

where $E_{\gamma}$ is the energy detected in the detector, $E_{\gamma 0}$ is the energy of the $\gamma$ ray in the rest frame of the nucleus, $\beta$ is the velocity of the nucleus emitting the $\gamma$ ray and $\theta$ is the angle between the velocity of the emitting nucleus and the $\gamma$ ray in the laboratory frame. From this we have a $\gamma$-ray peak width $\Delta E_{\gamma 0}$ of

$$
\left(\Delta E_{\gamma 0}\right)^{2}=\left(\frac{\partial E_{\gamma 0}}{\partial E_{\gamma}} \Delta E_{\gamma}\right)^{2}+\left(\frac{\partial E_{\gamma 0}}{\partial \beta} \Delta \beta\right)^{2}+\left(\frac{\partial E_{\gamma 0}}{\partial \theta} \Delta \theta\right)^{2} .
$$


This can be used to evaluate the performance of the PSA via the relation

$$
\cos \theta=\frac{\vec{v} \cdot \vec{r}}{|\vec{v}||\vec{r}|}
$$

where $\vec{v}$ is the recoil velocity as detected by VAMOS and $\vec{r}$ is the position vector of the first $\gamma$-ray interaction as given by $\gamma$ ray tracking. The method employed to determine the position ${ }_{655}$ resolution for six different AGATA crystals is to do Geant4 simulations that in a realistic way take into account all experimental contributions to the FWHM of the $\gamma$-ray peaks while varying the assumed position resolution of the PSA. The experimental FWHM of the $\gamma$-ray peak can then be used to interpolate the ${ }_{660}$ actual position resolution of the PSA.

In this case the experiment was a fusion-fission experiment populating, among other nuclei, ${ }^{98} \mathrm{Zr}$. A beam of ${ }^{238} \mathrm{U}$ impinged on a $10 \mu \mathrm{m}$ think ${ }^{9} \mathrm{Be}$ foil. The VAMOS was positioned at $28^{\circ}$ relative to the beam axis. Six AGATA detectors close to $112^{\circ}{ }_{66}$ relative to the recoil direction were used to sample the position resolution of the detectors in the array, as they had the largest Doppler Broadening, increasing the sensitivity to the position resolution. As all data was analyzed after $\gamma$-ray tracking it was the interaction used for Doppler Correction that determined ${ }_{670}$ which detector was studied. The FWHM of the $\gamma$-ray peaks were determined using Gaussian fits. An error $\Delta \beta / \beta=0.0045$ as deduced from the mass resolution of VAMOS gives a constant contribution to the FWHM of the $\gamma$-ray peak of $0.13 \%$.

The simulation took into account the energy loss in the tar- ${ }_{675}$ get and straggling as the product left the target as well as the acceptance of VAMOS. In the simulations a perfect knowledge of the recoil velocity was assumed $(\Delta \vec{v}=0)$. An intrinsic resolution of the AGATA detectors of $2.6 \mathrm{keV}$ at $1332 \mathrm{keV}$ was assumed for all detectors. Peak widths as a function of po- 680 sition resolution were determined for seven different position resolutions. As a baseline the experimentally determined position resolution from Söderström et al. [4] was used. The resolution as a function of energy determined by Söderström et al. [4] was scaled from 0.36 to 1.41 . This as the interaction point used for Doppler Correction can have any energy so there is no unambiguous position resolution extracted from a Doppler Broadened peak. However, from simulations it was calculated that the average position resolution for the interaction point used for the Doppler Correction was $4.3 \mathrm{~mm}$ FWHM when using the non-scaled function of Söerströ et al. [4]. For each assumed position resolution the FWHM of the simulated $\gamma$-ray peak for each detector was determined by a Gaussian fit. extra width coming from the error in recoil velocity was added quadratically. In figure 15 these values are shown with small symbols for detector 39 and 41 (which has the best and worst experimental position resolution, respectively). To each set of FWHM coming from the variation of position resolution a second degree polynomial function was fitted. Using the inverse of these function the position resolution of the individual detectors can be determined (see large symbols in figure 15. Note that in figure 15 the $\mathrm{x}$-axis is a scaling factor with the previously determined position resolution as base, i.e. 1 means the detector has a PSA performance au pair with what was previously measured. The six detectors used to sample the position resolution are located in the span 0.79-1.4, with five detectors larger than 1.08 with an weighted average of 1.15 .

\section{Angular Correlations in AGATA}

The use of AGATA for angular correlations measurements to determine the multipolarity of $\gamma$ decay has been investigated using source data. Two pairs of $\gamma-\gamma$ cascades from the decay of ${ }^{152} \mathrm{Eu}$ were used: The first pair was the $1408 \mathrm{keV}-121.8 \mathrm{keV}$ coincidence in ${ }^{152} \mathrm{Sm}$ de-exciting the $2_{1}^{-}$level at $1530 \mathrm{keV}$ to to the ground state via the $2_{1}^{+}$level at $121.8 \mathrm{keV}$. The second pair is the $244.7 \mathrm{keV}-121.8 \mathrm{keV}$ de-exciting the $4_{1}^{+}$level at $366.5 \mathrm{keV}$ and the $2_{1}^{+}$level, also in ${ }^{152} \mathrm{Sm}$.

The tracking algorithm identifies the first interaction point of each $\gamma$ ray and as the position of the source is known the angle between the $\gamma$ rays in the $1408 \mathrm{keV}-121.8 \mathrm{keV}$ pair and the $244.7 \mathrm{keV}-121.8 \mathrm{keV}$ pair could be determined and histogramed, see lower panel in figure 16 . The main features of the two pairs of $\gamma$ rays are similar. The cut at about 8 degrees is a result of the tracking algorithm, whereas for larger angles the geometry of AGATA as used for the source measurement dominates the shape of the spectra. The slower rise in intensity for the 244.7-121.8 keV cascade at low angles comes from the intrinsic difficulty to track two low-energy $\gamma$ rays emitted into a small solid angle. The angular correlation is then extracted by normalizing for geometrical effects and the already mentioned decrease in efficiency for two low-energy $\gamma$ rays absorbed close to each other. The normalization was created by tracking events consisting of the interaction points of two events each with a total energy corresponding to one of the $\gamma$-ray's in the cascade of interest concatenated into one event, thus generating pairs of $\gamma$ rays with the correct energies but with no angular correlation. From the tracked events the angle between the $\gamma$ rays was then again extracted. The resulting histograms for the two pairs of $\gamma$ rays are shown in the upper panel of figure 16 .

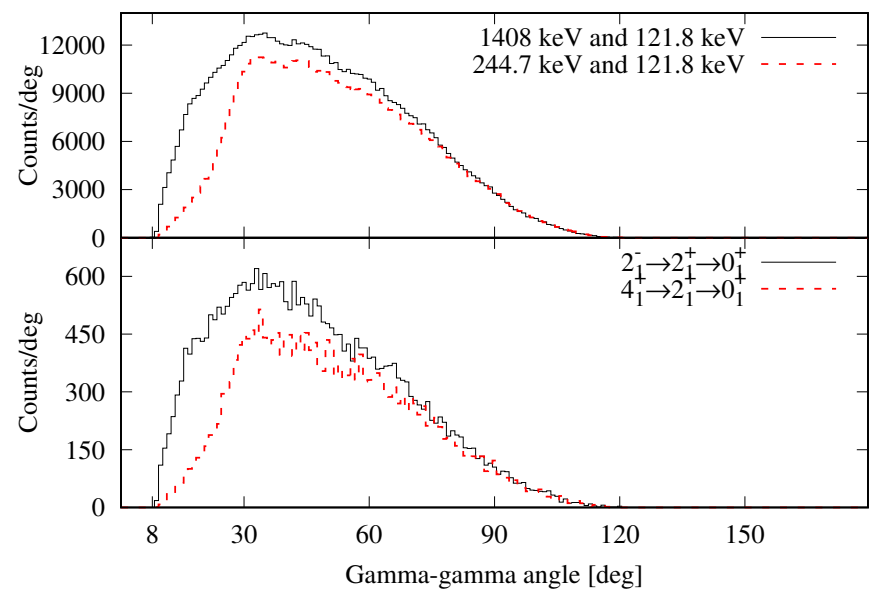

Figure 16: Histograms used for angular correlation measurements using AGATA. The lower panel shows the angle between the two correlated $\gamma$ rays detected in AGATA. The upper panel shows the angle between $\gamma$ rays from uncorrelated events concatenated before tracking. 
By dividing the histograms in the lower panel in figure 16 by the upper panel the histograms shown in figure 17 are created. The upper panel is for the $4_{1}^{+} \rightarrow 2_{1}^{+} \rightarrow 0_{1}^{+}$cascade, the lower panel for the $2_{1}^{-} \rightarrow 2_{1}^{+} \rightarrow 0_{1}^{+}$cascade. For each angular correlation two theoretical correlations have been fitted by a normalation coefficient, i.e. with mixing ratios kept to zero. Given in the legends are the resulting reduced $\chi^{2}$ for the fits using either a L2-L2 or L1-L2 cascade.

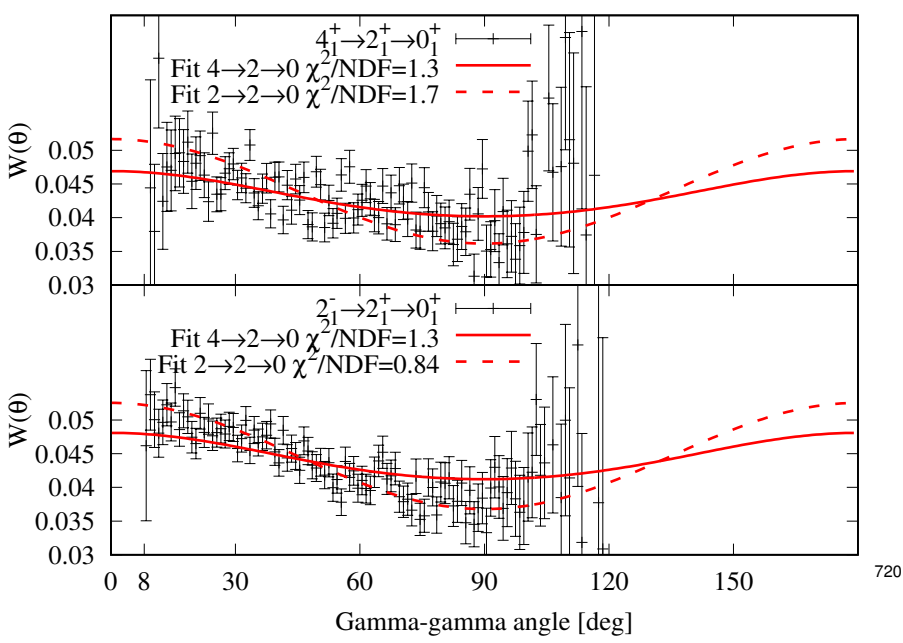

Figure 17: Gamma-gamma angular correlations measured with AGATA. The upper panel shows the angular correlation for the $4_{1}^{+} \rightarrow 2_{1}^{+} \rightarrow 0_{1}^{+}$pair of transitions. The lower panel is for the $2_{1}^{-} \rightarrow 2_{1}^{+} \rightarrow 0_{1}^{+}$transitions

The particularity of a $\gamma$-tracking array as compared to a classical multi-detector $\gamma$-ray spectrometer can be shown looking at the difference between using uncorrelated hits that are concatenated and tracked or tracked uncorrelated $\gamma$ rays concatenated 730 into events when constructing the normalization used to extract the angular correlations from the experimental correlations. In the top panel of figure 18 the histogram drawn with a black solid ne shows the distribution of theta angles between uncorrelated $\gamma$ rays concatenated after tracking. The histogram drawn with ${ }^{735}$ red dashed line shows the theta angle distribution if one instead concatenates uncorrelated events using the individual hits and then preforms the tracking. The bottom panel shows the resulting angular correlations using the two different methods of generating the normalisation.

\section{Conclusions and perspective}

The performance of AGATA installed at GANIL, coupled to the VAMOS has been characterized. The efficiency of AGATA, ${ }^{745}$ as a whole as well as for individual crystals, has been determined using both singles measurements and coincidence methods. It has been done both using AGATA as a standard array and as a $\gamma$-ray tracking array. A total efficiency for AGATA of $3.8(1) \%$ at $1332 \mathrm{keV}$ for the nominal geometry when using $\gamma-750$ AGATA is used as normal multi-detector array. It is also shown how the efficiency extracted from coincidence has to be corrected for angular correlation effects, as the increased probabil-

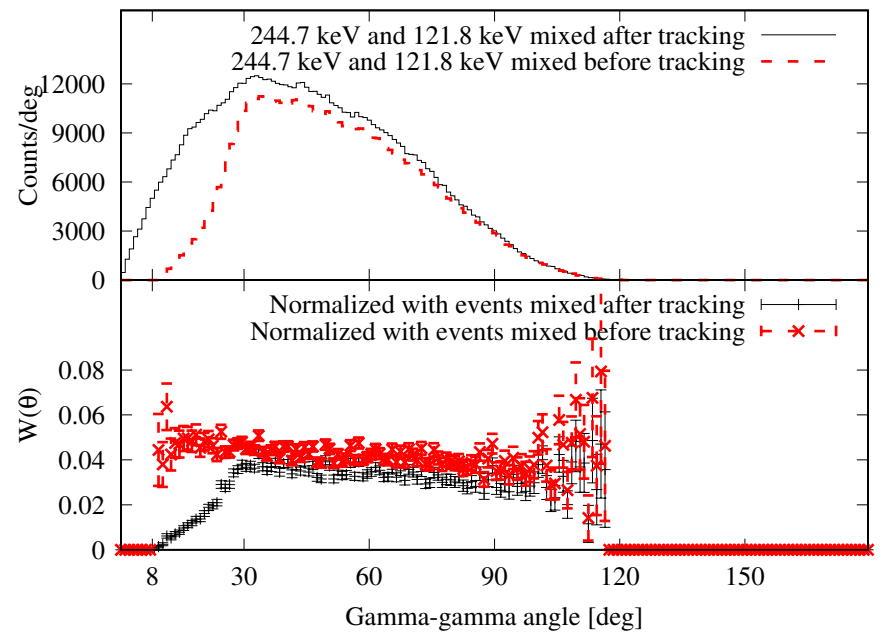

Figure 18: Normalization histograms and angular correlations for $4_{1}^{+} \rightarrow 2_{1}^{+} \rightarrow 0_{1}^{+}$using the "concatenate before tracking" and "concatenate after tracking" methods.

ity to emit parallel $\gamma$ rays combined with the clustering stage of $\gamma$-ray tracking generates a loss of efficiency that depends on the angle between the two $\gamma$ rays. This correction has to be made on top of the typical correction made for angular correlations effects.

As the AGATA detectors have been used in different campaigns the segments of some of the detectors are showing clear signs of neutron damages. Annealing procedures are complicated for these detectors and ingenious neutron damage correction procedures have been developed allowing an almost full recovery of the intrinsic energy resolution. The average central contact energy resolution for AGATA (beginning of 2016) is $2.57 \mathrm{keV}$ at $1332 \mathrm{keV}$ and for the segments $3.08 \mathrm{keV}$ at $1332 \mathrm{keV}$. Corresponding values before applying neutron damage correction are $3.08 \mathrm{keV}$ and $5.22 \mathrm{keV}$ for central contacts and segments, respectively. The neutron correction procedure is effective but at some point the detectors will need to be annealed. Such maintenance questions are clearly as important for the future of AGATA as the more appealing technical developments that can be made.

The position resolution given by the PSA for AGATA at 740 GANIL has been estimated using data from an experiment performed in the first half of the campaign. A sample of six of the AGATA crystals had there position resolution estimated. This was done by comparing experimental data with Monte Carlo simulation in which the position resolution was varied. It turns out that the average position resolution found was a factor of 1.16(5) larger than what was measured in a dedicated experiment [4].

As the number of crystals in AGATA increases the interest in using AGATA for angular correlations and distributions increases. Using a ${ }^{152} \mathrm{Eu}$ source angular correlations have been produced and methods to properly normalize for the combined effect of geometry and $\gamma$-ray tracking have been devised.

Finally, the AGATA detector system is performing very well, as proven by the physics results that have been produced. De- 
755 spite this, improvements in the PSA and further tuning of $\gamma$-ray tracking algorithms would be beneficial. The needed develop- 820 ment is related to better understanding of the details of the signal generation in the segmented detectors. This would in turn allow better handling multiple interactions in one segment and 760 removing the nonphysical clustering of interaction points. Such ${ }^{825}$ improvements should allow for an increased peak-to-total.

\section{Acknowledgments}

The authors would like to thank the AGATA collaboration and the GANIL technical staff. Gilbert Duchêne is thanked

765 for providing the in-beam data set used to extract the position 835 resolution of the pulse-shape analysis. Partially supported by the Ministry of Science, Spain, under the Grants BES-2012061407, SEV-2014-0398, FPA2017-84756-C4 and by the EU FEDER funds.

770 [1] S. Akkoyun, A. Algora, B. Alikhani, F. Ameil, G. de Angelis, L. Arnold, A. Astier, A. Ataç, Y. Aubert, C. Aufranc, A. Austin, S. Aydin, F. Azaiez, S. Badoer, D. Balabanski, D. Barrientos, G. Baulieu, R. Baumann, D. Bazzacco, F. Beck, T. Beck, P. Bednarczyk, M. Bellato, M. Bentley, G. Benzoni, R. Berthier, L. Berti, R. Beunard, G. L. Bianco, B. Birkenbach, P. Bizzeti, A. Bizzeti-Sona, F. L. Blanc, J. Blasco, N. Blasi, D. Bloor, C. Boiano, M. Borsato, D. Bortolato, A. Boston, H. Boston, P. Bourgault, P. Boutachkov, A. Bouty, A. Bracco, S. Brambilla, I. Brawn, A. Brondi, S. Broussard, B. Bruyneel, D. Bucurescu, ${ }_{850}$ I. Burrows, A. B Agata-advanced \{GAmma\} tracking array Nuclear Instruments and Methods in Physics Research Section A: Accelerators, Spectrometers, Detectors and Associated Equipment 668 (2012) $26-58$. doi:http://dx.doi.org/10.1016/j.nima.2011.11.081 URL http://www.sciencedirect.com/science/article/pii/ S0168900211021516

785 [2] I. Y. Lee, M. A. Deleplanque, K. Vetter, Developments in large gammaray detector arrays Reports on Progress in Physics 66 (7) (2003) 1095. URL http://stacks . iop.org/0034-4885/66/i=7/a=201

[3] F. Recchia, D. Bazzacco, E. Farnea, R. Venturelli, S. Aydin, G. Suli- ${ }_{860}$ man, C. Ur, Performance of an agata prototype detector estimated by compton-imaging techniques Nuclear Instruments and Methods in Physics Research Section A: Accelerators, Spectrometers, Detectors and Associated Equipment 604 (1) (2009) 60 - 63, pSD8. doi:https://doi.org/10.1016/j.nima.2009.01.079 URL http://www.sciencedirect.com/science/article/pii/ S0168900209001168

[4] P.-A. Söderström, F. Recchia, J. Nyberg, A. Al-Adili, A. Ataç, S. Aydin, D. Bazzacco, P. Bednarczyk, B. Birkenbach, D. Bortolato, A. Boston, H. Boston, B. Bruyneel, D. Bucurescu, E. Calore, S. Colosimo, F. Crespi, N. Dosme, J. Eberth, E. Farnea, F. Filmer, A. Gadea, A. Gottardo, X. Grave, J. Grebosz, R. Griffiths, M. Gulmini, T. Habermann, H. Hess, G. Jaworski, P. Jones, P. Joshi, D. Judson, R. Kempley, A. Khaplanov, E. Legay, D. Lersch, J. Ljungvall, A. Lopez-Martens, W. Meczynski, D. Mengoni, C. Michelagnoli, P. Molini, D. Napoli, R. Orlandi, G. Pascovici, A. Pullia, P. Reiter, E. Sahin, J. Smith, J. Strachan, D. Tonev, C. Unsworth, C. Ur, J. Valiente-Dobón, C. Veyssiere, 11 A. Wiens, Interaction position resolution simulations and in-beam and Methods in Physics Research Section A: Accelerators, Spectrometers, Detectors and Associated Equipment 638 (1) (2011) 96 - 109. doi:http://dx.doi.org/10.1016/j.nima.2011.02.089 URL http://www.sciencedirect.com/science/article/pii/ S016890021100489X

[5] A. Gadea, E. Farnea, J. Valiente-Dobn, B. Million, D. Mengoni, D. Bazzacco, F. Recchia, A. Dewald, T. Pissulla, W. Rother, G. de Angelis, A. Austin, S. Aydin, S. Badoer, M. Bellato, G. Benzoni, L. Berti, R. Beunard, B. Birkenbach, E. Bissiato, N. Blasi, C. Boiano, D. Bortolato, A. Bracco, S. Brambilla, B. Bruyneel, E. Calore, F. Camera, A. Capsoni, J. Chavas, P. Cocconi, S. Coelli, A. Colombo, D. Conventi, L. Costa,
L. Corradi, A. Corsi, A. Cortesi, F. Crespi, N. Dosme, J. Eberth, S. Fantinel, C. Fanin, E. Fioretto, C. Fransen, A. Giaz, A. Gottardo, X. Grave, J. Grebosz, R. Griffiths, E. Grodner, M. Gulmini, T. Habermann, C. He, H. Hess, R. Isocrate, J. Jolie, P. Jones, A. Latina, E. Legay, S. Lenzi, S. Leoni, F. Lelli, D. Lersch, S. Lunardi, G. Maron, R. Menegazzo, C. Michelagnoli, P. Molini, G. Montagnoli, D. Montanari, O. Mller, D. Napoli, M. Nicoletto, R. Nicolini, M. Ozille, G. Pascovici, R. Peghin, M. Pignanelli, V. Pucknell, A. Pullia, L. Ramina, G. Rampazzo, M. Rebeschini, P. Reiter, S. Riboldi, M. Rigato, C. R. Alvarez, D. Rosso, G. Salvato, J. Strachan, E. Sahin, F. Scarlassara, J. Simpson, A. Stefanini, O. Stezowski, F. Tomasi, N. Toniolo, A. Triossi, M. Turcato, C. Ur, V. Vandone, R. Venturelli, F. Veronese, C. Veyssiere, E. Viscione, O. Wieland, A. Wiens, F. Zocca, A. Zucchiatti, Conceptual design and infrastructure for the installation of the first agata sub-array at lnl Nuclear Instruments and Methods in Physics Research Section A: Accelerators, Spectrometers, Detectors and Associated Equipment 654 (1) (2011) 88 96. doi:https://doi.org/10.1016/j.nima.2011.06.004

URL http://www.sciencedirect.com/science/article/pii/ S0168900211011132

[6] C. Domingo-Pardo, D. Bazzacco, P. Doornenbal, E. Farnea, A. Gadea, J. Gerl, H. Wollersheim, Conceptual design and performance study for the first implementation of agata at the in-flight rib facility of gsi Nuclear Instruments and Methods in Physics Research Section A: Accelerators, Spectrometers, Detectors and Associated Equipment 694 (2012) 297 312. doi:https://doi.org/10.1016/j.nima.2012.08.039 URL http://www.sciencedirect.com/science/article/pii/ S0168900212009102

[7] N. Lalović, C. Louchart, C. Michelagnoli, R. Perez-Vidal, D. Ralet, J. Gerl, D. Rudolph, T. Arici, D. Bazzacco, E. Clément, A. Gadea, I. Kojouharov, A. Korichi, M. Labiche, J. Ljungvall, A. Lopez-Martens, J. Nyberg, N. Pietralla, S. Pietri, O. Stezowski, Performance of the \{AGATA\} $\gamma$-ray spectrometer in the prespec set-up at \{GSI\}. Nuclear Instruments and Methods in Physics Research Section A: Accelerators, Spectrometers, Detectors and Associated Equipment 806 (2016) 258 266. doi:http://dx.doi.org/10.1016/j.nima.2015.10.032 URL http://www.sciencedirect.com/science/article/pii/ S0168900215012395

[8] A. Korichi, T. Lauritsen, A. Wilson, J. Dudouet, E. Clément, N. Lalović, R. Perez-Vidal, S. Pietri, D. Ralet, O. Stézowski, Performance of a gamma-ray tracking array: Characterizing the agata array using a 60co source. Nuclear Instruments and Methods in Physics Research Section A: Accelerators, Spectrometers, Detectors and Associated Equipment 872 (2017) 80 - 86. doi:https://doi.org/10.1016/j.nima.2017.08.020

(1) URL http://www.sciencedirect.com/science/article/pii/ S0168900217308781

[9] E. Clément, C. Michelagnoli, G. de France, H. Li, A. Lemasson, C. B. Dejean, M. Beuzard, P. Bougault, J. Cacitti, J.-L. Foucher, G. Fremont, P. Gangnant, J. Goupil, C. Houarner, M. Jean, A. Lefevre, L. Legeard, F. Legruel, C. Maugeais, L. Ménager, N. Ménard, H. Munoz, M. Ozille, B. Raine, J. Ropert, F. Saillant, C. Spitaels, M. Tripon, P. Vallerand, G. Voltolini, W. Korten, M.-D. Salsac, C. Theisen, M. Zielińska, T. Joannem, M. Karolak, M. Kebbiri, A. Lotode, R. Touzery, C. Walter, A. Korichi, J. Ljungvall, A. Lopez-Martens, D. Ralet, N. Dosme, X. Grave, N. Karkour, X. Lafay, E. Legay, I. Kojouharov, C. DomingoPardo, A. Gadea, R. Pérez-Vidal, J. Civera, B. Birkenbach, J. Eberth, H. Hess, L. Lewandowski, P. Reiter, A. Nannini, G. D. Angelis, G. Jaworski, P. John, D. Napoli, J. Valiente-Dobón, D. Barrientos, D. Bortolato, G. Benzoni, A. Bracco, S. Brambilla, F. Camera, F. Crespi, S. Leoni, B. Million, A. Pullia, O. Wieland, D. Bazzacco, S. Lenzi, S. Lunardi, R. Menegazzo, D. Mengoni, F. Recchia, M. Bellato, R. Isocrate, F. E. Canet, F. Didierjean, G. Duchêne, R. Baumann, M. Brucker, E. Dangelser, M. Filliger, H. Friedmann, G. Gaudiot, J.-N. Grapton, H. Kocher, C. Mathieu, M.-H. Sigward, D. Thomas, S. Veeramootoo, J. Dudouet, O. Stézowski, C. Aufranc, Y. Aubert, M. Labiche, J. Simpson, I. Burrows, P. Coleman-Smith, A. Grant, I. Lazarus, P. Morrall, V. Pucknell, A. Boston, D. Judson, N. Lalović, J. Nyberg, J. Collado, V. González, I. Kuti, B. Nyakó, A. Maj, M. Rudigier, Conceptual design of the agata $1 \pi$ array at ganil Nuclear Instruments and Methods in Physics Research Section A: Accelerators, Spectrometers, Detectors and Associated Equipment 855 (Supplement C) (2017) 1 - 12. 
doi:https://doi.org/10.1016/j.nima.2017.02.063

II URL http://www.sciencedirect.com/science/article/pii/ S0168900217302590

[10] H. Savajols, Vamos: a variable mode high acceptance spectrometer Nuclear Physics A 654 (1) (1999) 1027c - 1032c.965 doi:http://dx.doi.org/10.1016/S0375-9474(00)88592-9 URL http://www.sciencedirect.com/science/article/pii/ S0375947400885929

[11] M. Rejmund, B. Lecornu, A. Navin, C. Schmitt, S. Damoy, O. Delaune, J. Enguerrand, G. Fremont, P. Gangnant, L. Gaudefroy, B. Jacquot,970 J. Pancin, S. Pullanhiotan, C. Spitaels, Performance of the improved larger acceptance spectrometer: Vamos++ Nuclear Instruments and Methods in Physics Research Section A: Accelerators, Spectrometers, Detectors and Associated Equipment 646 (1) (2011) 184 - 191. doi:http://dx.doi.org/10.1016/j.nima.2011.05.007 975

[13] J. Scheurer, M. Aiche, M. Aleonard, G. Barreau, F. Bourgine, D. Boivin, D. Cabaussel, J. Chemin, T. Doan, J. Goudour, M. Harston, A. Brondi,980 G. L. Rana, R. Moro, E. Vardaci, D. Curien, Improvements in the in-beam -ray spectroscopy provided by an ancillary detector coupled to a ge -spectrometer: the diamant-eurogam ii example Nuclear Instruments and Methods in Physics Research Section A: Accelerators, Spectrometers, Detectors and Associated Equipment 385 (3) (1997) 501 - 510.985 doi:https://doi org/10.1016/S0168-9002(96)01038-8

II URL http://www.sciencedirect.com/science/article/pii/ S0168900296010388

[14] J. Gál, G. Hegyesi, J. Molnár, B. Nyakó, G. Kalinka, J. Scheurer, M. Aléonard, J. Chemin, J. Pedroza, K. Juhász, V. Pucknell, The vxi 990 electronics of the diamant particle detector array Nuclear Instruments and Methods in Physics Research Section A: Accelerators, Spectrometers, Detectors and Associated Equipment 516 (2) (2004) 502 - 510. doi:https://doi.org/10.1016/j.nima.2003.08.158 URL http://www.sciencedirect.com/science/article/pii/395 S0168900203025075

[15] A. Wiens, H. Hess, B. Birkenbach, B. Bruyneel, J The \{AGATA\} triple cluster detector Nuclear Instruments and Methods in Physics Research Section A: Accelerators, Spectrometers, Detectors and Associated Equipment 618 (1-3) (2010) 223 - 2331000 doi:http://dx.doi.org/10.1016/j.nima.2010.02.102 URL http://www.sciencedirect.com/science/article/pii/ S0168900210003384

[16] S. Agostinelli, J. Allison, K. Amako, J. Apostolakis, H. Araujo, P. Arce, M. Asai, D. Axen, S. Banerjee, G. Barrand, F. Behner, L. Bellagamba1005 J. Boudreau, L. Broglia, A. Brunengo, H. Burkhardt, S. Chauvie, J. Chuma, R. Chytracek, G. Cooperman, G. Cosmo, P. Degtyarenko, A. Dell'Acqua, G. Depaola, D. Dietrich, R. Enami, A. Feliciello, C. Ferguson, H. Fesefeldt, G. Folger, F. Foppiano, A. Forti, S. Garelli, S. Giani, R. Giannitrapani, D. Gibin, J. G. Cadenas, I. Gonzlez101 G. G. Abril, G. Greeniaus, W. Greiner, V. Grichine, A. Grossheim, S. Guatelli, P. Gumplinger, R. Hamatsu, K. Hashimoto, H. Hasui, A. Heikkinen, A. Howard, V. Ivanchenko, A. Johnson, F. Jones, J. Kallenbach, N. Kanaya, M. Kawabata, Y. Kawabata, M. Kawaguti, S. Kelner, P. Kent, A. Kimura, T. Kodama, R. Kokoulin, M. Kossov, H. Kurashige, E. Lamanna, T. Lampn, V. Lara, V. Lefebure, F. Lei, M. Liendl, W. Lockman, F. Longo, S. Magni, M. Maire, E. Medernach, K. Minamimoto, P. M. de Freitas, Y. Morita, K. Murakami, M. Nagamatu, R. Nartallo, P. Nieminen, T. Nishimura, K. Ohtsubo, M. Okamura, S. O'Neale, Y. Oohata, K. Paech, J. Perl, A. Pfeiffer, M. Pia, F. Ranjard, A. Rybin, S. Sadilov, E. D. Salvo, G. Santin, T. Sasaki, N. Savvas, Y. Sawada, S. Scherer, S. Sei, V. Sirotenko, D. Smith, N. Starkov, H. Stoecker, J. Sulkimo, M. Takahata, S. Tanaka, E. Tcherniaev, E. S. Tehrani, M. Tropeano, P. Truscott, H. Uno, L. Urban, P. Urban, M. Verderi, A. Walkden, W. Wander, H. Weber, J. Wellisch, T. Wenaus, D. Williams, D. Wright, T. Yamada, H. Yoshida, D. Zschiesche, Geant4a simulation toolkit Nuclear Instruments and Methods in Physics Research Section A: Accelerators, Spectrometers, Detectors and Associated Equipment 506 (3) (2003) 250 - 303 doi:https://doi.org/10.1016/S0168-9002(03)01368-8
S0168900203013688

[17] E. Farnea, F. Recchia, D. Bazzacco, T. Kröll, Z. Podolyák, B. Quintana, A. Gadea, Conceptual design and monte carlo simulations of the \{AGATA\} array Nuclear Instruments and Methods in Physics Research Section A: Accelerators, Spectrometers, Detectors and Associated Equipment 621 (1-3) (2010) 331 - 343. doi:http://dx.doi.org/10.1016/j.nima.2010.04.043 URL http://www.sciencedirect.com/science/article/pii/ S0168900210008922

[18] D. Barrientos, M. Bellato, D. Bazzacco, D. Bortolato, P. Cocconi, A. Gadea, V. Gonzalez, M. Gulmini, R. Isocrate, D. Mengoni, A. Pullia, F. Recchia, D. Rosso, E. Sanchis, N. Toniolo, C. A. Ur, J. J. ValienteDobon, Performance of the fully digital FPGA-based front-end electronics for the GALILEO array IEEE Transactions on Nuclear Science 62 (6) (2015) 3134-3139. doi:10.1109/tns . 2015.2480243 URL https : //doi.org/10.1109\%2Ftns. 2015.2480243

[19] R. Venturelli, D. Bazzacco, Adaptive grid search as pulse shape analysis algorithm for $\gamma$-tracking and results, LNL Annual Report (2004) 220.

[20] A. Lopez-Martens, K. Hauschild, A. Korichi, J. Roccaz, J.-P. Thibaud, $\gamma$-ray tracking algorithms: a comparison Nuclear Instruments and Methods in Physics Research Section A: Accelerators, Spectrometers, Detectors and Associated Equipment 533 (3) (2004) 454 - 466. doi:https://doi.org/10.1016/j.nima.2004.06.154

URL http://www.sciencedirect.com/science/article/pii/ S0168900204014779

[21] B. Bruyneel, P. Reiter, A. Wiens, J Crosstalk properties of 36-fold segmented symmetric hexagonal $\{\mathrm{HPGe}\}$ detectors Nuclear Instruments and Methods in Physics Research Section A: Accelerators, Spectrometers, Detectors and Associated Equipment 599 (2-3) (2009) 196 - 208. doi:http://dx.doi.org/10.1016/j.nima.2008.11.011

11 URL http://www.sciencedirect.com/science/article/pii/ S0168900208015921

[22] B. Bruyneel, B. Birkenbach, J. Eberth, H. Hess, G. Pascovici, P. Reiter, A. Wiens, D. Bazzacco, E. Farnea, C. Michelagnoli, F. Recchia, Correction for hole trapping in agata detectors using pulse shape analysis, The European Physical Journal A 49 (5) (2013) 61.

[23] [link] URL https : //radware .phy . ornl.gov/main.html

[24] S. Paschalis, I. Lee, A. Macchiavelli, C. Campbell, M. Cromaz, S. Gros, J. Pavan, J. Qian, R. Clark, H. Crawford, D. Doering, P. Fallon, C. Lionberger, T. Loew, M. Petri, T. Stezelberger, S. Zimmermann, D. Radford, K. Lagergren, D. Weisshaar, R. Winkler, T. Glasmacher, J. Anderson, C. Beausang, The performance of the gamma-ray energy tracking in-beam nuclear array gretina Nuclear Instruments and Methods in Physics Research Section A: Accelerators, Spectrometers, Detectors and Associated Equipment 709 (2013) $44-55$. doi:https://doi.org/10.1016/j.nima.2013.01.009

URL http://www.sciencedirect.com/science/article/pii/ S0168900213000508

[25] A. Lopes-Martens, To be published.

[26] F. Recchia, D. Bazzacco, M. Bellato, D. Borolato, E. Farnea, D. Mengoni, Efficency and energy resolution of the agata demonstartor at high count rate., Tech. rep., LNL (2010). 